\title{
The Transit and Light Curve Modeller
}

\author{
Sz. Csizmadia ${ }^{\oplus \star}$ \\ Deutsches Zentrum für Luft- und Raumfahrt, Institut für Planetenforschung, D-12489 Berlin, Rutherfordstr 2, Germany
}

Accepted 2020 January 20. Received 2020 January 20; in original form 2018 May 15

\begin{abstract}
Transit and Light Curve Modeller (TLCM), a computer code with the purpose of analysing photometric time series of transits simultaneously with the out-of-transit light variations and radial velocity curves of transiting/eclipsing binary systems, is presented here. Joint light-curve and radial velocity fits are possible with it. The code is based on the combination of a genetic algorithm and simulated annealing. Binning, beaming, reflection, and ellipsoidal effects are included. Both objects may have their own luminosities and therefore one can use TLCM to analyse the eclipses of both exoplanet and welldetached binary systems. A simplified Rossiter-McLaughlin effect is included in the radial velocity fit, and drifts and offsets of different instruments can also be fitted. The impact of poorly known limb darkening on the Rossiter-McLaughlin effect is shortly studied. TLCM is able to manage red-noise effects via wavelet analysis. It is also possible to add parabolic or user-defined baselines and features to the code. I also predict that light variations due to beaming in some systems exhibiting radial velocity drift should be observed by, e.g. PLATO. The fit of the beaming effect is improved by invoking a physical description of the ellipsoidal effects, which has an impact on the modelling of the relativistic beaming; I also point out the difficulties that are stemming from the fact that beaming and first-order reflection effects have the same form of time dependence. Recipe is given, which describes how to analyse grazing transit events. The code is freely available.
\end{abstract}

Key words: techniques: radial velocities-brown dwarfs-planets: exoplanets-photometry: transiting exoplanets-stars: eclipsing.

\section{INTRODUCTION}

A transit of an exoplanet was observed in 1999 for the first time ${ }^{1}$ (planet of V376 Peg = HD 209458; Charbonneau et al. 2000; Henry et al. 2000). The first exoplanet discovered by the transit method was found in 2002 (V5157 Sgr = OGLE-TR-56; Konacki et al. 2003). Since then we could see an enormous increase in exoplanet detections: As of 2020 January, the up-to-date online catalogue of Schneider et al. (2011) contains 4173 planets and planetary candidates in 3096 planetary systems, exhibiting 678 multiple planetary systems, and, in addition, 2735 planetary candidates and unconfirmed objects in 2508 planetary systems (182 are multiple). Most of them are discovered by transit method or show transits (2749 exoplanets and 2428 planetary candidates; Schneider et al. 2011). One can expect that many exoplanets will be found in the future due to ongoing ground- [e.g. HATS (Bakos et al. 2009), WASP (Pollacco et al. 2006), NGTS (West et al. 2016), and KELT (Pepper et al. 2007)] and space-based [e.g. TESS (e.g. Sullivan et al. 2015, see also Sullivan et al. 2017), PLATO (Rauer et al. 2014), and maybe CHEOPS (Broeg et al. 2013)] missions.

Transiting exoplanets are similar to eclipsing binaries not only in the nature and cause of their light variation but also in their

\footnotetext{
^E-mail: szilard.csizmadia@dlr.de

${ }^{1}$ The planet itself was detected by radial velocities earlier; see Charbonneau et al. (2000).
}

importance: They provide the only way to determine the mass, radius, and the average density of an exoplanet without ambiguity, which are the most important parameters to establish the planetary nature and to study their internal structure, formation, and evolution. ${ }^{2}$ They also can be used for transit timing variation (TTV) studies, which means variation in the period that leads to deviations in the mid-time of the transit from a linear ephemeris, the transit depth variation, and transit duration variation. All of these variations are caused by changes in the orbital elements, which can have a gravitational origin (e.g. periastron precession (cf. Csizmadia, Hellard \& Smith 2019), gravitational interaction by a third body (moon, planet(s), brown dwarf, or other star in the system (e.g. Borkovits et al. 2011)), magnetic interaction between the star and the planet, mass-loss from the system via stellar wind or by the evaporation of the planet, etc.). The solution of the inverse TTV problem can be used to measure the masses of the exoplanets if there are at least two transiting objects in the same system, or it is

\footnotetext{
${ }^{2}$ Strictly speaking, this is true for double-lined spectroscopic and eclipsing/transiting binary systems only. Transiting planetary systems are onelined spectroscopic binaries, but the mass of the host star can be measured via different techniques, like isochrone fitting, asteroseismology, etc. RV measurements themselves provide only the $m \cdot \sin i$ product, where the inclination $i$ is not known in the absence of transits. Note that TTV masses seem to be systematically affected by unknown factors, maybe because of the mutual inclinations or unseen objects (e.g. Weiss \& Marcy 2014; Bruno et al. 2015) or poorly known stellar masses.
} 
suitable for detecting new, unseen objects in the system (Holman \& Murray 2005; Borkovits et al. 2011). The mid-transit time is usually determined by fitting a transit model to the individual transits (e.g. Csizmadia et al. 2010; Mallonn et al. 2015; Raetz et al. 2015, among others).

Transiting exoplanets can also be used to measure their own atmospheric characteristics and composition via monitoring the effective radius variation of the planet over wavelength (e.g. Sedaghati et al. 2016; Deming \& Seager 2017, and references therein). If the occultation of the planet is observed, then its surface temperature, eccentricity, and argument of periastron can be estimated with a high accuracy (e.g. Huber, Czesla \& Schmitt 2017, among other examples). Areas with different albedos and surface temperatures resemble stellar spots on the star's surface because they affect the light curve (LC) similarly, and hence can be detected by applying the usual stellar spot-modelling techniques to the surface (atmosphere) of the exoplanet (e.g. Winn 2010). Transiting exoplanets also give information about their formation and evolution processes via measurements of the Rossiter-McLaughlin effect (RM effect) during transits (e.g. Albrecht 2012).

All of these studies require the analysis of the information content via fitting a model to the transit LCs. Appropriate models were recently developed (Mandel \& Agol 2002), or were imported from the previous binary star tools simplified appropriately (e.g. Gimenez 2006a; Soutworth 2013). However, as Wilson (1994) pointed out a model is not a code. The user-friendly, convenient, reliable, and extensive applications of these models to real LCs - the LC fitting itself - require a code, a choice and implementation of the optimization method, error estimation, visualization of the results, and detailed tests of the code. I mean two kinds of tests: the first one is for discovering and repairing bugs and programing errors (i.e. validation of the code), and the second kind is to establish the numerical features and properties of the fitting procedures. However, a final judgement of the reliability of the results is based solely on the user astronomer, since the role of experience is not taken by any kind of artificial intelligence yet.

In this paper I present the IDL-based code TLCM in detail, which is widely used for transit fits. The abbreviation TLCM stands for Transit and Light Curve Modeller. It runs under $\mathrm{IDL}^{3}$ or freely available $\mathrm{GDL}^{4}$ and its aim is to carry out the fit of the transit + occultation + outof-transit variation + radial velocity (RV) model to the observed LC, to find the best agreement between model and observations, and to estimate the uncertainties of the fitted parameters. A module callable from PYTHON will be available too.

Although TLCM has been used in several CoRoT publications (e.g. for CoRoT-6b, 8b, 9b, 13b, 15b, 17b, 21b, 23b) and many times it was used to (cross-)check the results obtained by other codes (e.g. CoRoT-5b, 7b, 11b, 18b, 19b, 20b), a complete description has never been published. TLCM has a long list of further applications, including Kepler-90's seven transiting planet systems (Cabrera et al. 2014) and other K2-objects (Eigmüller et al. 2016, 2017; Smith et al. 2017, 2019, Korth et al. 2019) among other space-based and groundbased photometric results (Rauer et al. 2009, 2010; Bordé et al. 2010; Cabrera et al. 2010, 2015; Csizmadia et al. 2010, 2011, 2013, 2015; Deeg et al. 2010; Fridlund et al. 2010; Gandolfi et al. 2010, 2013; Bouchy et al. 2011; Hébrard et al. 2011; Guenther et al. 2012; Ollivier et al. 2012; Paetzold et al. 2012; Rouan et al. 2012; Klagyivik et al.

\footnotetext{
${ }^{3}$ Interactive data language.

${ }^{4} \mathrm{Gnu}$ data language.
}

2016). The agreement between TLCM and JKTEBOP was found to be excellent in Soutworth (2012) for the checked cases.

The interested reader can find more descriptions of codes of others in Deeg (2018) and in the references therein, used in the field of exoplanets. As a comparison of others, one can say that TLCM does not model multiplanet systems simultaneously contrary to EXOFASTv2 (Eastman, J. D. et al. 2019, subm. to PASP), although it was used to model the transits of the seven planets in Kepler-90, modelling each planet's transits separately (Cabrera et al. 2014). TLCM takes the red-noise effects into account while exonailer does not.

Deeg (2018) listed 47 codes in total, which are designed to analyse exoplanets. 23 out of 47 (49 per cent) are designed for modelling transmission spectra, stellar spectra, planetary atmospheres, orbital dynamics, stellar spot analysis, out-of-transit variations, validation by checking possible blend scenarios, predicting limb-darkening coefficients, or doing data visualization. Four eclipsing binary codes are mentioned: ellc and JKTEBOP are able to fit LCs of eclipsing binaries, while PHOEBE 2.0 and Binary Maker 3 are able to fit jointly RV and LC. I would extend this list with the important Wilson-Devinney code (Wilson \& Devinney 1971, Wilson 1979, 1990, 2008), which is also a joint RV+LC fitter. JKTEBOP is extensively used for transiting exoplanet LC fits by John Southworth (Soutworth 2014); the others are rarely applied to exoplanets. Deeg (2018) lists nine further codes that perform only LC fits - three of the nine are for analysing multitransit system LCs or exoring transits. Seven further codes are for only RV analysis. He mentions only three codes that are able to fit jointly the RV and LC of a transiting host star: EXOFAST, exonailer, and TLCM. I extend this list with pyaneti (Barragán, Gandolfi \& Antoniciello 2019). TLCM is able to fit:

(i) either the LC only,

(ii) or the LC and RV of the primary simultaneously,

(iii) or the LC of the system and the RVs of both components simultaneously

(iv) or the LC +1 or $2 \mathrm{RVs}$ and the out-of-transit variations with red noise management.

The ability to fit two RV curves can be important to extend the range of applicability to well-detached SB2 systems of stars, because then the spectral lines and RV curves of both components might be measured. There exists other codes used but a detailed description was not published so far and therefore they are not listed in Deeg (2018).

TLCM is able to fit simultaneously the following phenomena for one transiting planet:

Fitting the above is complemented with a wavelet-based red noise model in the LC part and with the determination of the absolute parameters of the system (if the primary's temperature is known) and allowing the possibility for the user to add self-defined functions that cause baseline variations beyond second order.

\section{THE MODEL}

\subsection{Inputs and notations}

Before describing the model in detail, the prerequisites should be discussed.

The input photometric data should be either in the format of [time in days, normalized intensity, uncertainty, exposure time in seconds] or [photometric phase, normalized intensity, uncertainty, exposure time in seconds]. In the latter case period $=1.0$ should be set and 
in this case the calculated stellar density is not a valid result - the code sends a warning message for such a case. The RV data should be in the format [time, RV, uncertainty of RV, instrument identifier number], and one has to fill one file for the primary and, if the secondary's RV curve is available, another file for the secondary. If the RV curve is to be fitted too, then the photometric data file must contain time and not phase. The time should be in heliocentric or barycentric Julian Date and the same type of time-scale should be used for the photometric and RV curves.

Throughout this paper, I call the primary object the host star and the secondary object the planet. However, the code is able to fit well-detached $\left(a / R_{\mathrm{S}}>10\right.$ - for spherical star-planet systems no such limit exists) eclipsing binary LCs too. In those cases, the primary and secondary objects are stars and the star behind the other at photometric phase zero is called the primary.

The forward problem (i.e. calculating the LC for a given set of parameters) needs a set of parameters, which are the inputs of the code. This parameter set is summarized in Table 1 .

The conjunction parameter in Table 1 is defined as

$b^{\prime}=\frac{a\left(1-e^{2}\right) \cos i}{R_{\mathrm{s}}(1+e \sin \omega)}$.

For the fit and for the forward problem, I use $b^{\prime}$ instead of $i$ inclination. $i=0^{\circ}$ corresponds to a pole-on orbit, and $i=90^{\circ}$ to an edge-on orbit. The definition is that the inclination is the angle between the line of sight and the orbital angular momentum vector. The reason for using $b^{\prime}$ instead of $i$ is that $b^{\prime}$ can be better constrained in the fit because if a transit is observed one knows a priori that $0<b^{\prime}<1+k$ should hold and there is no sense to search outside this region; the use of inclination means more complex constraints.

Notice that in circular orbits $b^{\prime}=b=\delta_{\min }$, the conjunction parameter is equal to the impact parameter $b$ and the minimum skyprojected distance $\delta_{\min }$, but in eccentric orbits this is not the case; see equations (4) and (14).

I also make the note that $b^{\prime}$ is not restricted to have only positive values - except if the user use such a restriction when one sets such a box prior in the input config file. When such a prior is not used by the user, then positive and negative conjunction and impact parameters are allowed, meaning that inclination values below and over 90 degrees are also allowed. However, for central transits such a missing constrain may yield a bias in the impact parameter and thus in the inclination estimation because the median of the conjunction/impact parameter may artificially be shifted closer to zero.

The following combinations of the linear and quadratic limbdarkening coefficients are used in TLCM:

$u_{+, i}=u_{\text {linear }, i}+u_{\text {quadratic }, i}$,

$u_{-, i}=u_{\text {linear }, i}-u_{\text {quadratic }, i}$

where $i$ can be either the star (s) or the planet (p). It was found by numerical experiments in Brown et al. (2001) and later was confirmed by analytical calculations by Pál (2008) that these combinations of limb-darkening coefficients provide better performance because they are less sensitive to degeneracies and intercorrelations between the parameters. Note that I am not aware of a similar study about such useful combinations for other limb-darkening laws; therefore, such a study could be timely.

\subsection{The sky-projected mutual distance}

TLCM consists of a model and an optimizer. The heart of the model is the subroutines of Mandel \& Agol (2002), which provides the theoretically expected flux of a spherical, unspotted star transited by a spherical, absolutely dark (flux $l_{\text {planet }}=0$ ) planet. (The present version of the code uses the faster, newer version of the model, presented by Eastman et al. 2013.) That routine allows the user the choice between linear and quadratic limb-darkening laws. The output is a flux value, with a normalization constraint that the flux should be 1.0 at phase 0.25 (cf. Section 2.10). This can be compared to the observed flux value during the optimization process. The inputs of the Mandel \& Agol (2002) routines should be: the ratio of the radii of the planet and the star, $k=R_{\mathrm{p}} / R_{\mathrm{S}}$ (of course, $k>0.0$ holds). The planet can also be bigger than the star, e.g. the case of a white dwarf and a larger super-Earth - or $k>1$ in the case of occultation. If any iterational step sets negative $k$, TLCM calculates the transit function with its absolute value but it adds the resultant transit to the LC with an opposite sign, i.e. causing a 'positive hump' to attempt to avoid the Lucy-Sweeney bias in the radius ratio in small transits at low signal-to-noise $(\mathrm{S} / \mathrm{N})$ ratios, the scaled semimajor axis, $a / R_{\mathrm{S}}\left(a / R_{\mathrm{S}}>1+k\right.$ holds - when this condition is violated, TLCM takes $a / R_{\mathrm{S}}=1+k$ in circular orbits or $\mathrm{a} / R_{\mathrm{S}}=(1+k) /(1-\mathrm{e})$ in elliptic orbits, the limb-darkening coefficients, ${ }^{5}$ and the mutual sky-projected distance between the apparent stellar centre and the apparent planetary centre, denoted by $\delta$.

The sky-projected distance between the centres of the components, as is well known, is given by the following equations (e.g. Russell 1912; Milone \& Kallrath 2009, equation 3.1.10, Gimenez 2006b, equation 19):

$$
\begin{aligned}
n_{P} & =\frac{2 \pi}{P} \\
E-e \sin E & =M=n_{P}(t-\tau)=n_{P}\left(t-T_{0}\right)+M_{0} \\
\tan \frac{v}{2} & =\sqrt{\frac{1+e}{1-e}} \tan \frac{E}{2}
\end{aligned}
$$

(for $M_{0}$ see equation 12) and

$\delta=\frac{\left(a / R_{\mathrm{s}}\right) \times\left(1-e^{2}\right)}{1+e \cos v} \sqrt{1-\sin ^{2} i \sin ^{2}(v+\omega),}$

where $n_{P}, E$, and $v$ are the mean motion, and the eccentric and true anomalies, respectively. For the calculation of periastron passage time $\tau$, see Section 2.3. Note that transit community uses the notation $\delta$ or sometimes $\delta_{T}$ for the approximate transit depth as $\delta_{T}=\left(R_{\text {planet }} / R_{\text {star }}\right)^{2}$, but here it is used for the mutual sky-projected distance.

The transformation of the true space orbit into projected orbit is done via (expressed in stellar radius units, cf. Milone \& Kallrath 2009, equation 3.1.8):

$$
\begin{aligned}
r & =\frac{\left(a / R_{\mathrm{s}}\right) \cdot\left(1-e^{2}\right)}{1+e \cdot \cos v} \\
X & =r \cdot \sin (v+\omega) \cdot \sin i \\
Y & =r(\cos (v+\omega) \sin \Omega+\sin (v+\omega) \cos i \cos \Omega) \\
Z & =r(\cos (v+\omega) \cos \Omega-\sin (v+\omega) \cos i \sin \Omega)
\end{aligned}
$$

The sky-projected view of the system is given by $Y$ and $Z$ and it is visualized in Fig. 1. In other words, the mutual distance is $\delta=$ $\sqrt{Y^{2}+Z^{2}}$ and by substitution one can see that this is independent of $\Omega$. The axis $X$ is oriented towards the observer. The origin of this $X Y Z$ coordinate system is in the centre of the host star.

\footnotetext{
${ }^{5}$ These are calculated easily from the $u_{+}$and $u_{-}$combinations.
} 
Table 1. Description of parameters used in TLCM. LDC stands for limb-darkening coefficient.

\begin{tabular}{ll}
\hline Notation & \\
\hline$T_{0}$ & Transit epoch. \\
$P:$ & Period. \\
$a / R_{1}$ & $a$ : semimajor axis, $R_{1}$ is the stellar radius. \\
$k=R_{1} / R_{2}$ & Planet-to-star radius ratio. \\
$\sqrt{e} \sin \omega$ & $e:$ eccentricity, $\omega:$ argument of periastron. \\
$\sqrt{e} \cos \omega$ & Conjunction parameter. \\
$b$ & Combinations of LDCs for the star. \\
$u_{+, s}, u_{-, s}$ & Combinations of LDCs for the planet. \\
$u_{+, p}, u_{-, p}$ & Third light. \\
$l_{3}$ & Longitude of the node in degrees. \\
$\Omega$ &
\end{tabular}

$\begin{array}{ll}A_{1}, A_{2} & \text { Albedos of the star and the planet. } \\ \sigma_{w}, \sigma_{r} & \text { White noise and the red noise factors. } \\ p_{0}, p_{1}, p_{2} & \text { Constant, linear, and quadratic terms of } \\ & \text { a fitted parabolic flux change. } \\ t_{\text {parabola }} & \text { Centre time of the parabola. } \\ h & \text { Height correction-parameter. } \\ N_{T} & \text { Time-resolution factor. } \\ & \\ V_{\gamma} & \text { RV of the system's common centre of mass to us. } \\ K_{s} & \text { RV half-amplitude of the primary. } \\ r v_{\text {offset }} & \text { RV offset between different spectrographs. } \\ d_{1}, d_{2} & \text { Linear and quadratic terms for RV drifts. } \\ q=M_{2} / M_{1} & \text { The mass ratio of the two objects. }\end{array}$

$T_{\text {eff }} \quad$ Effective temperature of the star.

$f \quad$ Surface brightness ratio of the components.

Z Metallicity of the star.

$\lambda \quad$ The effective wavelength of the observations.

$V \sin I_{1,2} \quad$ Projected equatorial rotational speed.

$\lambda_{1,2} \quad$ The projected angle between the stellar rotational axis and the orbital planes.

$N_{\text {init }} \quad$ Number of individuals in a population.

$T_{\mathrm{SA}} \quad$ Control parameter of the Simulated Annealing (SA).

$n_{\max } \quad$ The maximum number of iterations.

in SA and in MCMC.
The epoch is the mid-transit time of any un/observed transit.

In days or $=1$ in case of phase units (see the main text).

The code expects and fits their ratio, which is called the scaled semimajor axis.

$R_{2}$ is the planetary radius. The code expects and fits $\mathrm{k}$.

The code expects and fits these combinations. ${ }^{a}$

See the main text for further discussion.

$-$

See equation (2) and paragraph after that for more information about it. See Section 2.10 and equation (51) for more information about it. It has no impact on the light-curve fit; it rotates only the sky view of the system (see Fig. 1).

$-$

$-$

$-$

It is measured in phase units.

See Section 2.9 for more details.

It is for taking into account the smoothing of extremely long exposures (like in the $\sim 29$ min exposure time of Kepler's long-cadence data. Section 2.11).

In $\mathrm{km} \mathrm{s}^{-1}$. It is positive when the system's distance is increasing from us.

In $\mathrm{km} \mathrm{s}^{-1}$.

It is given in $\mathrm{km} \mathrm{s}^{-1}$.

Their units are $\mathrm{km} \mathrm{s}^{-1} /$ day and $\mathrm{km} \mathrm{s}^{-1} / \mathrm{day}^{2}$.

$M_{i}$ stands for the components' mass. Only used for double-lined spectroscopic systems (SB2) where the spectral lines of both components can be seen or for systems where the beaming or the ellipsoidal effect is strong enough that beaming+ellipsoidal effects can be simultaneously fitted. For SB1 (one-line systems) or systems without RV data, it is not used, except in case of ellipsoidal effect is needed. It is kept fixed during the fitting procedure.

Passband dependent.

Fixed during fitting procedure.

In nanometres.

In $\mathrm{km} \mathrm{s}^{-1}$.

In degrees.

See Section 4.1.

See Section 4.2.

See Section 4.2.

Note. ${ }^{a}$ Version 95 and later ones fit $\sqrt{e} \sin \omega$ and $\sqrt{e} \cos \omega$. Version 94 and earlier ones fitted $e \sin \omega$ and $e \cos \omega$. See Eastman at al. (2013) and Albrecht (2012) about the complications that different parametrizations can introduce.

The minimum value of $\delta$ during transit is called the impact parameter, which for circular orbits is

$b=a / R_{\mathrm{s}} \cos i$

while for eccentric orbits

$b=\frac{\left(a / R_{\mathrm{s}}\right) \cdot\left(1-e^{2}\right)}{1+e \cos v_{0}} \sqrt{1-\sin ^{2} i \sin ^{2}\left(v_{0}+\omega\right)}$.

The exact value of $v_{0}$ is calculated in the next subsection. Using the approximation $v_{0} \approx 90^{\circ}-\omega$ and substituting this into equation (4), one gets the conjunction parameter (equation 1). $v_{0}$ is the true anomaly when $\delta$ has its minimum value during transit corresponding to the deepest transit depth - and its exact value is calculated in the subsequent subsection. Notice that for occultations $v_{0} \approx 270^{\circ}-\omega$, so $b$ can be different for transits and occultations in non-circular orbits.

The values of - or at least constraints on $-e$ and $\omega$ can be obtained from the observations from the secondary eclipse (or occultations), $\mathrm{RV}$ measurements, or phase curves. When this is not the case, I use equation (6) to calculate the sky-projected distance.

Occultations (secondary transits) in CoRoT LCs can be only tentatively detectable (Parviainen, Deeg \& Belmonte 2013) and also rarely observable in Kepler LCs (Coughlin \& López-Morales 2012). Remarkable exceptions are CoRoT-1b whose occultations can be observed both by a satellite (Snellen, de Mooij \& Albrecht 2009; Parviainen et al. 2013) and from the ground (Alonso et al. 2009) and CoRoT-2b (Alonso et al. 2010 from the ground in NIR and Parviainen et al. 2013 by CoRoT) as well as CoRoT-6b, 11b, and the 


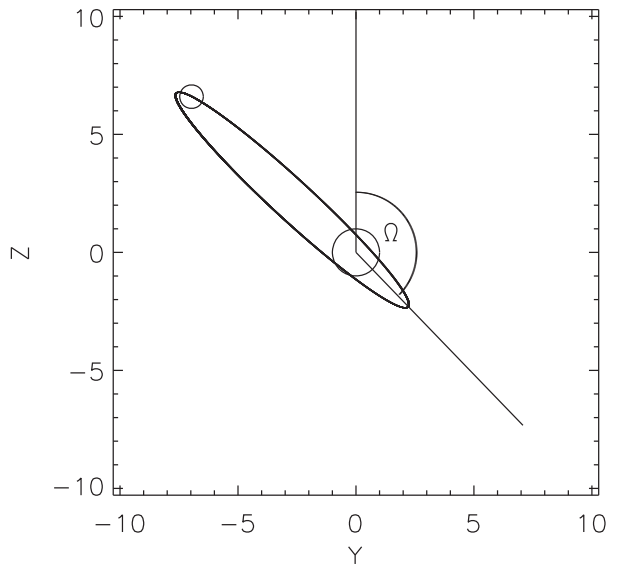

Figure 1. Illustration of the meaning of orbital element $\Omega$, the longitude of the node. Positive $Z$-direction is north on the tangential plane of the sky at the place of the star, and positive $Y$-direction is in the direction of east. The example shows an eccentric orbit with different impact parameters during occultation and transit.

brown dwarf CoRoT-15b (Parviainen et al. 2013). For well-studied secondary eclipse detections in the Kepler data, see Angerhausen, DeLarme \& Morse (2015).

RV measurements also do not always constrain the orbit with the desired precision (because many of the targets are in the fainter regime or the number of observed RV data points is insufficient; see e.g. Zakamska, Pan \& Ford 2011). For example, Hébrard et al. (2011) reported $e=0.33 \pm 0.1$ for CoRoT-16b and Gandolfi et al. (2010) reported $e<0.6$ for CoRoT-11b. In the case of CoRot-16b, this eccentricity error causes approximately 19 per cent relative error in the determination of the $a / R_{1}$ ratio. GJ $1214 \mathrm{~b}$ has only an upper limit for eccentricity too $(e<0.27$; Charbonneau et al. 2009) and the list of poorly measured eccentricities could be continued (cf. with the list of 231 eccentricities of Bonomo et al. 2017). Note that the transit length itself does not constrain $a / R_{1}$ because transit length also depends on the radius ratio $k$, impact parameter $b, e$, and $\omega$ themselves (see equation 70). The well-known correlation between $a / R_{1}$ and $b^{\prime}$ (see e.g. Csizmadia et al. 2011) will then be further affected by the uncertainties of $e$. In addition, when the $\mathrm{S} / \mathrm{N}$ ratio is very small, then it is not possible to determine $a / R_{1}$ even for circular orbits from the LC itself with the desired accuracy; one needs an additional constraint like stellar density to get an idea about $a / R_{1}$ (Barros et al. 2014). This constraint is based on the known relationship derivable from Kepler's third law:

$\rho_{\text {star }}=\frac{3 \pi}{G P^{2}\left(1+M_{\mathrm{p}} / M_{\mathrm{s}}\right)}\left(\frac{a}{R_{1}}\right)^{3}$.

In many applications, $M_{\mathrm{p}} / M_{\mathrm{s}} \approx 0$, when this is not true (stellar or brown dwarf companion, for instance), the mass ratio should be estimated, e.g. from the available RV curves of the primary and the secondary or from the ellipsoidal effect. When the stellar density is not available, ${ }^{6}$ then I need some other procedure.

${ }^{6}$ The main problem is the density of single stars - densities of SB2 eclipsing systems can be obtained in a model-independent way with a high accuracy. Mean stellar density can be learned from asteroseismology, but most of the stars are too faint, or the time sampling too sparse or observational window is too short to utilize it. However, it is a good prospective for PLATO (Rauer et al. 2014). Spectroscopically measured $\log g$ and $T_{\text {eff }}$ of the star can locate the place of the star in HRD yielding its mean density, but this method is

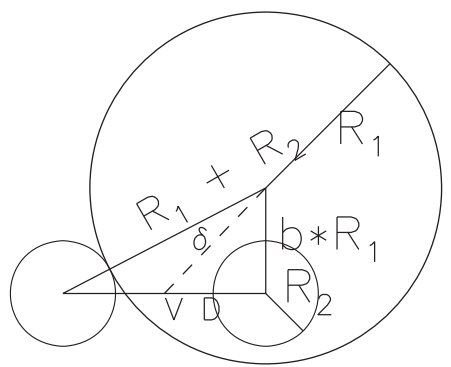

Figure 2. Estimation of sky-projected mutual distance $(\delta)$ of the centres of the primary and secondary objects under the assumption that during transit duration $D$ the tangential velocity $V$ of the secondary relative to the primary is constant. $R_{j} \mathrm{~s}$ are the radii of the two spherical objects; $b$ is the impact parameter. The position of the planet on the line connecting the first contact and mid-transit point is found by linear interpolation. This yields one side of a right-angled triangle, and its another side is the constant $b \cdot R_{1}$. The hypotenuse is the searched $\delta$ distance that can be obtained by applying Pythagoras' theorem.

For those cases where $e / \omega$ values are not available, another kind of calculation was introduced for the sky-projected distance (Csizmadia \& Pasternacki 2013), which is based on a linear interpolation between the mid-transit and the first contact as (Fig. 2; see also Csizmadia \& Pasternacki 2013):

$\delta\left(t_{j}\right)=\sqrt{b^{2}+\left((1+k)^{2}-b^{2}\right)\left(\frac{\varphi\left(t_{j}\right)}{\Delta \varphi_{14}}\right)^{2}}$,

where $\varphi$ is the photometric phase:

$\varphi_{j}=\frac{t_{j}-T_{0}}{P}$,

where $t_{j}$ is the mid-time of the $j$ th observation and $\Delta \varphi_{14}=(t-$ $\left.T_{0}\right) / \Delta T_{14}, \Delta T_{14}$ being the full duration of the transit. Its advantage is that it contains only photometrically measurable or fittable parameters and it requires no spectroscopic or other data input. Our tests showed that this formulation works quite well even for close-in planets too. If the scaled semimajor axis is smaller than 3 , or one needs to model the out-of-transit variations, then one has to use the precise equation (4) instead of (6).

After setting the sky-projected mutual distance for every time when one has a measurement, the code calls the subroutines of Mandel \& Agol (2002), and calculates the flux $(F)$ of the star for these time-moments $t_{i}$ ( $i$ is the index for the measurements):

$\operatorname{Tr}\left(t_{i}\right)=M A\left(k, u_{+s}, u_{-, s}, \delta\left(a / R_{s}, e, \omega, i, P, \tau\right)\right)$
$O c\left(t_{i}\right)=M A\left(k^{-1}, u_{+, p}, u_{-, p}, \delta\left(a / R_{p}, e, \omega, i, P, \tau\right)\right)$

or

$\operatorname{Tr}\left(t_{i}\right)=M A\left(k, u_{+, s}, u_{-, s}, \ldots, \delta\left(b, k, T_{0}, \Delta T_{14}\right)\right)$

$O c\left(t_{i}\right)=M A\left(k^{-1}, u_{+, p}, u_{-, p}, \ldots, \delta\left(b, k, T_{0}, \Delta T_{14}\right)\right)$.

Here, $M A$ denotes the Mandel-Agol equations and subroutines, whose outputs are the theoretically expected flux values. $T r$ stands for the transit and denotes the fractional light loss of the primary, and $O c$ does the same for the secondary during occultation event.

severely affected by uncertainties of the measured $\log g$ values, input stellar physics, convection theory, and degeneracies in the isochrones. 


\subsection{Further note on the calculation of sky-projected distance}

In case of eccentric, inclined orbits, the commonly reported midtimes of transits and occultations can differ from the conjunction times by as much as some minutes. This is much more than the $\sim 20$ s timing error in mid-transit time observations (e.g. Csizmadia et al. 2010). Readers can see Appendix $C$ to get the definition of conjunction and the period variations caused by proper motion, which moves the conjunction point. This makes the correct interpretation of LCs and transit timings difficult. It is explained below why this difference occurs and how one can take it into account.

Fig. 3 illustrates the geometry of the impact and the conjunction parameters. When the orbit is circular, $e=0$ and $\omega=90^{\circ}$ are set, then the impact and conjunction parameters are identical to each other. In case of an eccentric orbit, the periastron passage time $\tau$, the photometric epoch $T_{0}$, and the true anomaly at the middle of the transit $v_{0}$ can be linked to each other via the following set of equations:

$\frac{T_{0}-\tau}{P}=\frac{E_{0}-e \sin E_{0}}{2 \pi}=\frac{M_{0}}{2 \pi}$

$\tan \frac{E_{0}}{2}=\sqrt{\frac{1-e}{1+e}} \tan \frac{v_{0}}{2}$.

The true anomaly at the moment of the epoch can be decomposed as

$v_{0}=90^{\circ}-\omega+\theta$.

$v_{0}, E_{0}$, and $M_{0}$ are the true, eccentric, and mean anomalies when $\delta$ reaches its minimum at transit (for occultation, one has to add $180^{\circ}$ to the r.h.s. of equation 13).

In some other systems, we can also expect significant deviations between conjunction and mid-transit times: e.g. the referee of this paper pointed out that he found $175 \mathrm{~s}$ difference for HATS-41.

Actually, this may affect the transit timing analysis of eccentric systems and it can be an additional reason why the TTV masses seem to differ systematically from RV masses, but a detailed exploration of the topic is beyond the scope of this paper.

The appearance of the angle $\theta$ is the consequence of an inclined, eccentric orbit and usually it has a value of just a few arcminutes. A recipe of how to calculate it is given by the eclipsing binary star community (Martynov 1973; Gimenez \& Garcia-Pelayo 1983). According to them, the first and second derivatives of equation (4) and the substitution of equation (13) yield

$\tan \theta=\mp \frac{e \cos \omega \cos ^{2} i}{\cos \theta \sin ^{2} i \pm e \sin \omega}$.

The upper signs are valid for transit and the lower signs are for occultation. This is solved iteratively starting from $\theta=0$. (The correction is apparently built-in into the subroutines of Gimenez 2006a, but not into Mandel \& Agol 2002 because it expects directly the sky-projected distance). Conjunction occurs when the planet crosses the North-South meridian $(Y=0)$ and usually this moment does not coincide with the moment when the centres of the two objects are projected closest on the sky to each other (the eclipse is the deepest).

Usually, the transit epoch is known with much higher accuracy than the RV-based periastron passage time; therefore, I apply this correction in the following way, which also means a reduction in the number of free parameters: When the RV curve is fitted too, then one may adjust $T_{0}$ and set

$\tau=T_{0}-\frac{M_{0} \times P}{2 \pi}$.
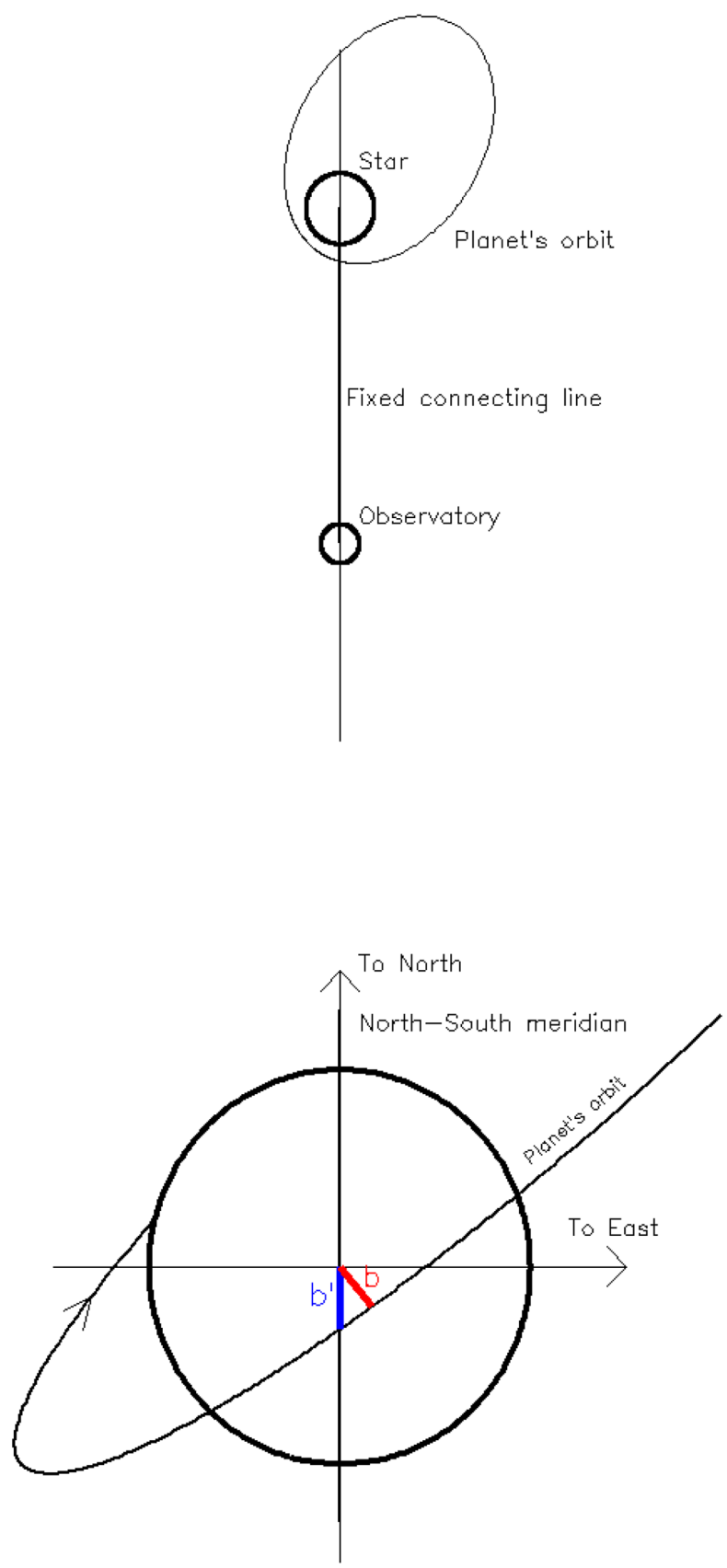

Figure 3. Upper figure: illustration of the star, the planetary orbit, and the place of the observatory. In principle, the line connecting the stellar centre and the observatory is not changing (or any change due to RV, proper motion or observatory's motion in Solar system can be corrected out in known ways). When transit (or occultation) occurs the planet crosses this fixed line and we time the planet's position always at the same place. Note that orbital elements, hence the orbit's shape and orientation (e.g. Csizmadia et al. 2019), can change. Lower panel: Meaning of impact parameter $b$ (red line) as the smallest sky-projected distance of the centres of the host star and the planet, which, in eccentric orbits, does not necessarily lie on the central meridian. However, the conjunction parameter $b^{\prime}$ (blue line) is always on that. Notice that the orbit can rotate due to general relativistic and tidal effects and therefore the red line is continuously changing its position. 


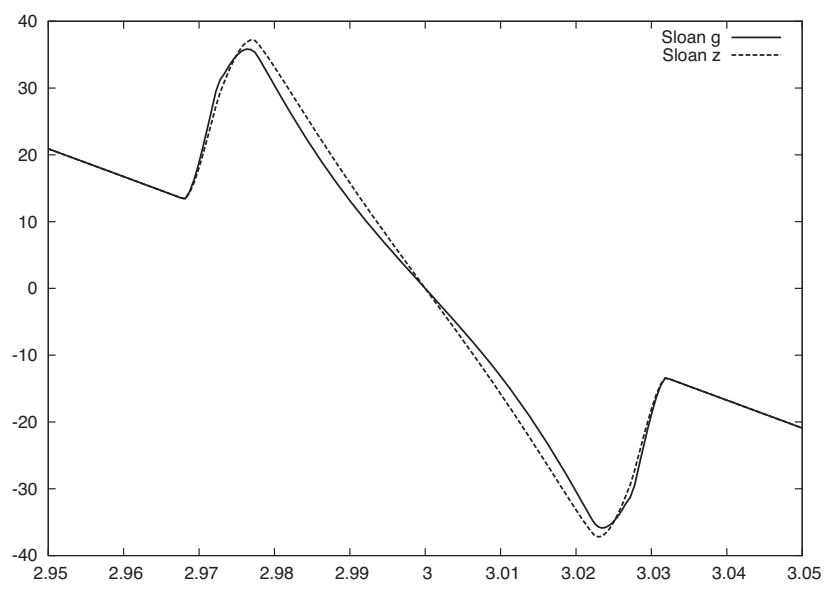

Figure 4. The RV variation during the transit of a 3 d-orbital period hot Jupiter (abscissa: time in days, ordinate: $\mathrm{RV}$ in $\mathrm{m} \mathrm{s}^{-1}$ ). Relevant parameters used to produce this plot were: $a / R_{\mathrm{S}}=14, k=0.1115, b=0.6, e=0, K_{1}=$ $0.2 \mathrm{~km} \mathrm{~s}^{-1}, V \sin I_{\mathrm{s}}=18 \mathrm{~km} \mathrm{~s}^{-1}, \beta_{1}=0^{\circ}$, and $V_{\gamma}=0 \mathrm{~km} \mathrm{~s}^{-1}$. The two curves correspond to two different limb-darkening coefficients of the same event, one to the Sloan $g^{\prime}$ band and one to the Sloan $z^{\prime}$ band. The maximum difference is ca. $3 \mathrm{~m} \mathrm{~s}^{-1}$, while the amplitude of the RM effect - relative to the RV curve due to pure orbital motion - is about $19 \mathrm{~m} \mathrm{~s}^{-1}$. This means that the two curves differ by about 16 percent. Such a difference is much smaller than the measurement error today and therefore one can neglect it. Consequently, a mean limb-darkening coefficient can be used for modelling the RM effect. (The same is not true for the LCs; Csizmadia et al. 2013.) However, higher precision RV measurements coming in the future, e.g. by ESPRESSO, or smaller RV amplitudes due to smaller/more distant planets will require a study of this issue. The effect of stellar spots on Rossiter measurements should be also studied.

This procedure is done before TLCM starts to calculate the skyprojected distance via equation (4).

The code is able to fit in the time domain or in the phase domain. The photometric phase is simply linked to the mean anomaly via

$M=2 \pi \varphi+M_{0}$,

where $M_{0}=E_{0}-e \sin E_{0}$ is the mean anomaly at the epoch (recall, I count the photometric phase from the moment of mid-transit).

\subsection{RV curves}

It is desirable to be able to fit the LC only (this can be important for planetary candidate ranking to save telescope time before one starts the RV measurements), and also to be able to fit jointly the RV and photometry, which is valuable because there are more constraints and fewer degrees of freedom. The present RV model also includes the RM effect. Our RV treatment for the primary is

$$
\begin{aligned}
V_{\mathrm{rad}, 1}\left(t_{j}, l\right)= & V_{\gamma}+K_{1}\left(e \cos \omega+\cos \left(v_{j}+\omega\right)\right) \\
& +\Sigma_{l=1}^{n} r v_{\mathrm{offset}}\left(t_{j}, l\right)+d_{1}\left(t_{j}-\tau\right)+d_{2}\left(t_{j}-\tau\right)^{2} \\
& +(V \sin i)_{1} Y\left(\lambda_{1}\right)(1-T r)
\end{aligned}
$$

and for the secondary

$$
\begin{aligned}
V_{\mathrm{rad}, 2}\left(t_{j}, l\right)= & V_{\gamma}-\frac{K_{1}}{q}\left(e \cos \omega+\cos \left(v_{j}+\omega\right)\right) \\
& +\Sigma_{l=1}^{n} r v_{\text {offset }}\left(t_{j}, l\right)+d_{1}\left(t_{j}-\tau\right)+d_{2}\left(t_{j}-\tau\right)^{2} \\
& -(V \sin i)_{2} Y\left(\lambda_{2}\right)(1-O c)
\end{aligned}
$$

and the minus sign in the second term of equation (18) comes from the fact that the periastron of the secondary object is at $\omega+180^{\circ}$.

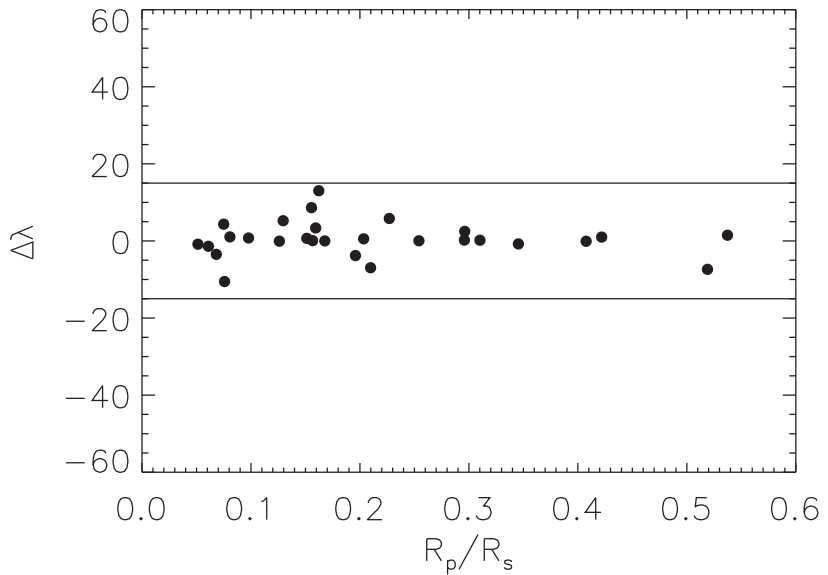

Figure 5. Study of the accuracy of the small-planet approximation in the $\mathrm{RM}$ effect, radius ratio $\left(R_{\mathrm{p}} / R_{\mathrm{S}}\right)$ versus accuracy of $\Delta \lambda . \Delta \lambda$ is the difference in degrees between the synthetic input value and the resultant value of the model carried out with the small-planet approximation. The two horizontal lines denote the $\pm 15^{\circ}$ limits and they can be taken as limits of the accuracy of this approximation. For this test, a uniform, $2 \mathrm{~m} \mathrm{~s}^{-1}$ scatter in the RV curves was assumed as observational errors and every point had a different $a / R_{\text {star }}$, $V \sin I$ (between 3 and $28 \mathrm{~km} \mathrm{~s}^{-1}$ ), $\lambda$, radius ratio, and limb darkening. At the present level of precision, this accuracy seems to be enough - however, a full analytic RM model will be implemented in future versions.

The angle $\lambda_{i}$ can be different for the two components and it means 'the angle between the position of the stellar rotational axes projected on the plane of the sky with that of the pole of the orbit' (Gimenez 2006b; he denoted it by $\beta_{i}$ ). In my treatment, angle $\Omega$ should be replaced by $\lambda_{i}$ in the expression of $Y$ (Section 2.2). While the first three lines in equations (17) and (18) are standard expressions, the last term, describing the RM effect, is a simplified expression for it. In TLCM, a small-planet approximation is used for simplicity to take the RM effect into account. The $T r$ and $O c$ functions are the transit and occultation functions introduced by equations (8) and (9), and I allow that for the RM effect these functions may have their own limb-darkening coefficients. This is because the spectroscopic observations may be carried out in a different wavelength region to the photometric measurements; therefore, RM effect and photometry may have different limb darkenings. Notice that limb darkening is changing over wavelength. Typically, Rossiter-McLaughlin measurements are done using a wide wavelength range of spectra in order to increase of the lines used, which is absolutely necessary to reach the desired accuracy. Therefore, here we would need an integral or an appropriately weighted limb-darkening coefficient. I found with numerical experiments (Fig. 4) that if the Sloan $g^{\prime}$-band limb-darkening coefficients are used instead of the $\mathrm{z}^{\prime}$ band, then the maximal difference between the two RV curves during transit is about 16 per cent for a typical hot Jupiter scenario (for stars it can be higher). This kind of measurements can cover such a big wavelength range. Therefore, the code asks for an appropriately weighted limbdarkening set for the fit of the RM effect or alternatively fits it.

To check the range of applicability of the RM effect treatment, I compared this model to numerically calculated RV curves. For the numerical model, the star was divided to 5 million surface elements and I performed several simulations with different input parameters: radius ratio, limb darkening, inclination, $V \sin I$, and $\lambda$ angle. I found that if one allows the limb-darkening coefficients to vary in the RM fit, then TLCM can recover the RM parameter $\lambda$ within \pm 15 deg (Fig. 5) - probably the free limb darkening is able 
to compensate some of the effects neglected in the small-planet approximation.

$r v_{\text {offset }}\left(t_{j}, l\right)$ is a zero-point shift to take into account that sometimes one has RV measurements from different instruments, where $l$ is the index of the instrument used for the RV measurement at $t_{j}$. The drift, characterized by $d_{1}$ and $d_{2}$, can be due to unknown companions as, for instance, it was applied to interpret the RV curve of K2-99b by Smith et al. (2017).

When one fits the RV and LC together without red noise, the $\chi^{2}$ values of them are simply summed up. No scaling factor is applied to increase the importance of one curve over the other.

\subsection{Emission of the secondary}

As is known, Jupiter and Saturn in our own Solar system emit more energy than they receive from the Sun; this is due to their slow contraction under self-gravity, which produces heat. Although this radiation is more prominent in infrared, however, being a blackbody radiation, it is present at all wavelengths. Notice that observations of several exoplanet occultations (see the references in Section 2.2) show that many hot Jupiters exhibit a flux of $\sim 10^{-4}$ part of the star, that is $100 \mathrm{ppm}$, while PLATO will have $34 \mathrm{ppm} / \mathrm{h}^{1 / 2}$ photometric precision (Rauer et al. 2014), and Kepler and in some cases CoRoT have already reached this or even better precision for bright host stars. In addition, if the secondary is a brown dwarf or a star, then a considerable amount of isotropic emission can be expected from the object. Therefore, the secondary emits flux in this model. It is denoted by $n$ and it is

$n=\left(\frac{R_{\mathrm{p}}}{R_{\mathrm{s}}}\right)^{2} f$,

where $f$ is the surface brightness ratio of the two objects at the effective wavelength of the observations; bolometrically, it would be

$f_{\text {bol }}=\left(\frac{T_{2}}{T_{1}}\right)^{4}$

but in case of blackbody approximation:

$f_{\text {passband }}=\frac{\mathrm{e}^{14388 \mu \mathrm{K} / \lambda / T_{1}}-1}{\mathrm{e}^{14388 \mu \mathrm{K} / \lambda / T_{2}}-1}$.

Here, $\lambda$ is the effective wavelength of the filter or response function (in microns) used in the observations, and $T_{i}$ stands for the temperatures of the components (in Kelvins). The constant 14388 is the $h c / k$, being the Planck constant, speed of light, and the Boltzmann constant expressed in micron - Kelvin. For real spectra, the ratio $f$ can be quite different from the blackbody approximation.

TLCM calculates $f$ from the primary's temperature and from the value of $f$. In case of space-based CoRoT, Kepler and TESS data the code requires to specify the name of the satellite and via equations (35) and (36) it calculates a more precise effective temperature value of the secondary from $T_{\mathrm{eff}, 1}$ and from the fitted or prescribed $f$, using theoretical stellar spectra (see Section 2.6.2).

Thus, the code also calculates $T_{2}$ for the secondary object, but it should be noted while $f$ is exact, $T_{2}$ might be approximate.

\subsection{Beaming effect}

The beaming effect or Doppler boosting is a special relativistic effect and means that the light of an isotropically emitting object (in the rest frame) will focus its light in the direction of motion (in the observer's frame). The effect is described and characterized in Loeb \& Gaudi (2003) and its first successful application was to
CoRoT-3b by Mazeh \& Faigler (2010). Since then, the effect has been observed in quite a few systems.

For the primary the beaming effect is taken as

$B_{1}\left(t_{j}\right)=-\alpha_{b, 1} V_{\mathrm{rad}, 1}\left(K_{1}, e, \omega, d_{1}, d_{2}, v\left(t_{j}\right)\right) / c$

and for the secondary, I take

$B_{2}\left(t_{j}\right)=n \cdot \alpha_{b, 2} V_{\mathrm{rad}, 2}\left(K_{1} / q, e, \omega, d_{1}, d_{2}, v\left(t_{j}\right)\right) / c$,

where $q=M_{2} / M_{1}$ is the mass ratio and functions $V_{\text {rad, } 1}$ and $V_{\text {rad, } 2}$ are given by similar expressions to equations (17) and (18). $\alpha_{i}$ s are the spectral indices. The flux variation caused by beaming consists of two parts. The first part is a periodic effect with the orbital period caused by orbital motion. The second part is due to the systematic velocity and RV drift, which should cause observable, but small changes in the mean flux level of the star since its distance is changing. Using the drift value of K2-99b (Smith et al. 2017), one expects $10 \mathrm{ppm} \mathrm{yr}^{-1}$ brightening. Notice that such an effect might be observable by PLATO, because one can average the LC over a long time (up to several months) to detect such effects. Its detection requires the control of long-term systematics: star-spots, stellar variability, rotation, and instrumental drifts. If these can be taken into account, then such an effect can be used to detect unseen companions too. ${ }^{7}$

\subsubsection{Spectral index: blackbody approximation}

For computing the spectral index factor $\alpha$, the inputs are the temperature of the primary and the surface brightness ratio of the two objects; therefore, the secondary's temperature is estimated with the assumption that both the objects are black bodies. One can verify that in this case:

$T_{2}=\frac{14388 \mu \mathrm{K}}{\lambda \ln \left(1+\frac{1}{f}\left(\mathrm{e}^{14388 \mu \mathrm{K} / \lambda / T_{1}}-1\right)\right)}$.

According to Loeb \& Gaudi (2003), the spectral indices are

$\alpha_{b, i}=\frac{14388 \mu \mathrm{Ke}^{14388 \mu \mathrm{K} / \lambda T_{i}}}{\lambda\left(\mathrm{e}^{14388 \mu \mathrm{K} / \lambda / T_{i}}-1\right)}$

and the wavelength should be substituted in microns (the code expects it in nanometres and automatically converts it to microns).

\subsubsection{Spectral index: approximation by real stellar atmospheres}

However, when the blackbody approximation was applied to some of the real systems observed by K2 it was found in Eigmüller et al. (2018) that this is not precise enough. Therefore, a passbandintegrated beaming coefficient for the photometry of CoRoT, Kepler, and $\mathrm{K} 2$ is used as was done in Bloemen et al. (2011) too. This is as follows:

$\alpha_{b, i, \bmod }=\frac{\int T(\lambda) S\left(\lambda, T_{i}, \log g,[\mathrm{Fe} / \mathrm{H}]\right) \alpha_{b, i}\left(\lambda, T_{i}\right) \mathrm{d} \lambda}{\int \operatorname{Tr}(\lambda) S\left(\lambda, T_{i}, \log g,[\mathrm{Fe} / \mathrm{H}]\right) \mathrm{d} \lambda}$

where $T(\lambda)$ is the transmission or the sensitivity function of the used instruments, and $S$ is the template spectrum from Kurucz models as is provided by Munari et al. (2005).

Since the modified beaming factors are functions of the stellar log $g$ and metallicity, it is time-consuming to re-calculate these factors in every iterational step when the temperature is changing. Therefore,

${ }^{7}$ The brightness change due to change of the distance to the star caused by the systematic velocity of the system is negligible, less than $0.1 \mathrm{ppm} \mathrm{yr}^{-1}$. 
I plotted these modified $\alpha_{b}$ factors against temperature, averaged for all metallicity and $\log g$ and the following approximate formulae are used instead of equation (26) with

$x_{i}=T_{i} / 5775 K$.

Below $T=20000 \mathrm{~K}$ for CoRoT:

$$
\begin{aligned}
\alpha_{b, i, \text { mod }}-5= & 43.1405-30.4439 \frac{x_{i} \mathrm{e}^{1.48512 x_{i}}}{\mathrm{e}^{x_{i}}-1}+37.3894 x_{i}^{2} \\
& -5.824404 x_{i}^{3}+2.301 x_{i}^{4}
\end{aligned}
$$

and for Kepler:

$$
\begin{aligned}
\alpha_{b, i, \bmod }-5= & 31.938-24.4348 \frac{x_{i} \mathrm{e}^{1.35289 x_{i}}}{\mathrm{e}^{x_{i}}-1}+15.5532 x_{i}^{2} \\
& +2.34049 \exp \left(x_{i}\right)
\end{aligned}
$$

while for TESS:

$$
\begin{aligned}
\alpha_{b, i, \text { mod }}-5= & 2.47645-31.5295 \frac{x_{i} \mathrm{e}^{-3.22304 x_{i}}}{\mathrm{e}^{x_{i}}-1}-0.385524 x_{i}^{2} \\
& +0.122321 \exp \left(x_{i}\right) .
\end{aligned}
$$

Over $20000 \mathrm{~K}$ one can use for CoRoT:

$$
\begin{aligned}
\alpha_{b, i, \text { mod }}-5= & -1.52933-0.792617 \frac{x_{i} \mathrm{e}^{0.868462 x_{i}}}{\mathrm{e}^{x_{i}}-1} \\
& +0.00073304 x_{i}^{3}-0.0110599 x_{i}^{2}
\end{aligned}
$$

and for Kepler

$\alpha_{b, i, \bmod }-5=-1.97886-0.647688 \frac{x_{i} \mathrm{e}^{0.868333 x_{i}}}{\mathrm{e}^{x_{i}}-1}$.

For TESS, I needed two more temperature ranges for appropriate fits. Between 20000 and $34000 \mathrm{~K}$ :

$\alpha_{b, i, \bmod }-5=-1.91355-0.735441 \frac{x_{i} \mathrm{e}^{0.85822 x_{i}}}{\mathrm{e}^{x_{i}}-1}$

while over $34000 \mathrm{~K}$ :

$\alpha_{b, i, \bmod }-5=-5.21913+0.391325 x_{i}-0.0254576 x_{i}^{2}$.

However, it was found to be more accurate than this approximations if I interpolate a table of what I calculated for a grid of temperatures, $\log g$, and metallicity with a grid of $250 \mathrm{~K}$ in temperature, 0.5 in $\log g$, and 0.5 in metallicity. Using this approach, the computational speed is considerably faster than the integral of equation (26) performed in every iteration step and the code still produces good results (Eigmüller et al. 2018).

Equations (28)-(34) and Fig. 6 also show that cooler stars have larger spectral indices, i.e. they produce bigger beaming effects at the same RV. Thus, cooler stars may exhibit more evident beaming curves.

Similarly, the calculation of $T_{2}$ from $T_{1}$, and the intensity ratio $f$ from the wavelength, is also based on the same spectral library of Munari et al. (2005) and it is given by the following algorithm. The intensity of the primary is

$I_{1}=a+b x_{1}+c x_{1}^{2}+d x_{1}^{3}+f x_{1}^{4}+g \mathrm{e}^{x_{1}}$

and then

$\log T_{\text {eff }, 2}=g_{1}+h \log f I_{1}+i \log ^{2} f I_{1}+j \log ^{3} f I_{1}+k \log ^{4} f I_{1}$.

Appropriate coefficients for the three main exoplanet satellites are in Table 2

When only the LC is fitted, the beaming - when observable - is a useful parameter to estimate the RV amplitude. When RV and LC are



Figure 6. Dependence of spectral index on effective temperature for the Kepler passband. The scatter in some temperature ranges is due to different metallicities and $\log g \mathrm{~s}$ of the template stars.

simultaneously fitted, the code uses the RV amplitude to calculate the beaming.

\subsection{Ellipsoidal variability}

When a planetary-sized or bigger celestial object rotates and/or it is subject to tidal interaction, its equilibrium shape is not spherical anymore. When the rotation and the tidal deformations are relatively small, it is enough to characterize this variation by the light changes due to a two- or three-axial ellipsoid. Since I assume that the flattening of this ellipsoid is small, the transit/eclipse phenomena can still be calculated via a spherical model. TLCM ignores this discrepancy, which should be small as long as the ellipsoidal variation is small.

The flux variation caused by ellipsoidal effects is denoted by $E_{i}$. For the primary object, I use the equation of

$E_{i}\left(t_{k}\right)=\Sigma_{j=1}^{J} a_{i j} P_{j}\left(\cos u_{k} \sin i\right)(a / r)^{j+2} \times[1, n]$

while

$u_{k}=v\left(t_{k}\right)+\omega-90.0^{\circ}$.

The vector $[1, n]$ describes the luminosity of the components: 1 should be used for the primary and $n$ for the secondary. The terms $a_{i j}$, according to Kopal's (1959) calculations, are related to the mass ratio, the semimajor axis, the radius of the objects, limb and gravity darkening, and internal density distributions. This latter factor is not well known for exoplanets so far; for stars we have some estimations (the $\log k_{2}$ parameter; for a review, see Torres, Andersen \& Giménez 2010). Usually, $a_{i j}$ s are expected to be small and they are polluting the reflection effect and hence may be intercorrelated with it. $a_{i 2}$ should be the dominating factor in the above expression. The $\sin i$ factor provides that pole-on orbits will show no ellipsoidal variations (as one expects). The $a_{12}$ factor can be used to estimate the ellipsoidal mass ratio of the two objects:

$$
\begin{aligned}
q_{\mathrm{ell}}= & \left(\frac{a}{R_{1}}\right)^{3} \\
& \times \frac{5 a_{12}\left(6-2 u_{1}-7 u_{2}\right)}{2\left(15+u_{1}+2 u_{2}\right)\left(1+0.25 \tau\left(\frac{5}{1+k_{2}}-1\right)\right) \sin ^{2} i\left(1+2 k_{2}\right)} .
\end{aligned}
$$

Here, $\tau$ is the correction for the gravity darkening (see Kopal et al. 1959 ) and $k_{2}$ is the so-called apsidal motion constant. To get its value, 
Table 2. Coefficients for equations (35) and (36). Metalicity and surface gravity dependence is not taken into account.

\begin{tabular}{|c|c|c|c|c|c|c|c|}
\hline Satellite & a & $\mathrm{b}$ & $\mathrm{c}$ & $\mathrm{d}$ & $\mathrm{f}$ & $\mathrm{g}$ & Validity range \\
\hline Kepler & $-9.38987 \times 10^{-8}$ & $6.8287 \times 10^{-8}$ & $9.51496 \times 10^{-8}$ & $-1.52921 \times 10^{-8}$ & $1.60095 \times 10^{-9}$ & $-6.57164 \times 10^{-10}$ & $3500<T_{\text {eff }}<47500 \mathrm{~K}$ \\
\hline TESS & $g_{1}$ & $\mathrm{~h}$ & $\mathrm{i}$ & $\mathrm{j}$ & $\mathrm{k}$ & & \\
\hline CoRoT & -82.4477 & -55.4495 & -12.9088 & -1.30535 & -0.0487586 & & $3500<\mathrm{T}_{\mathrm{eff}}<47500 \mathrm{~K}$ \\
\hline Kepler & -100.957 & -66.2159 & -15.2311 & -1.52606 & -0.0565664 & & $3500<\mathrm{T}_{\text {eff }}<47500 \mathrm{~K}$ \\
\hline
\end{tabular}

I estimate the absolute stellar parameters (Section 3), and the tables of Claret (2004) are used to estimate $k_{2}$. Note that apsidal motion constant of a star is of the order of $0 . . .0 .05$ usually, so it has a very small impact on the final results.

When TLCM fits the ellipsoidal effect and simultaneously the RV curves of both objects and/or the beaming effect of both components, then a different $q$ is used to estimate the mass ratio in equation (18) to get the RV amplitude of the secondary. A penalty function (the absolute value of the difference of $q_{\mathrm{ell}}-q$ ) is added to the minimized $\chi^{2}$ or $-\log L$ to reach an agreement. Also, a penalty value of $\mid a_{\mathrm{RV}} / R_{\mathrm{S}}$ $-a / R_{1} \mid$ is also added in this case, $a / R_{1}$ is the fitted scaled semimajor axis, related to the transit duration, while $a_{\mathrm{RV}}$ is the true semimajor axis obtained via

$a_{\mathrm{RV}}=\frac{K_{1} P(1+q)}{2 \pi q \sin i} \sqrt{1-e^{2}}$

and $R_{\mathrm{S}}$ is the absolute stellar radius estimated from $T_{\text {eff }}$, metallicity, and mean density of the star (Section 3). The advantage of this constraint during beaming analysis is that it makes the analysis selfconsistent.

\subsection{Reflection effect}

The reflected light can be minimal, but still observable with highprecision space photometry in exoplanetary systems. It can also be prominent in well-detached binaries; therefore, TLCM manages it.

The primary's reflection effect is simply characterized by

$$
R_{1}\left(t_{j}\right)=f A_{1} \frac{1+\cos u \sin i}{2}\left(\frac{k}{a / R_{1}} \frac{1-e^{2}}{1+e \cos v\left(t_{j}\right)}\right)^{2} .
$$

Here $A_{1}$ is the albedo of the primary. For the secondary, one has a more complicated expression, which is valid for giant planets (see Kane \& Gelino 2011, and references therein):

$$
\begin{aligned}
R_{2}\left(t_{j}\right)= & \left(A_{2}+0.2 \frac{\mathrm{e}^{r-1}-\mathrm{e}^{1-r}}{\mathrm{e}^{r-1}+\mathrm{e}^{1-r}}\right) . \\
& \times G(\alpha) \cdot\left(\frac{k}{a / R_{1}} \frac{1-e^{2}}{1+e \cos v\left(t_{j}\right)}\right)^{2}
\end{aligned}
$$

The function $G(\alpha)$ is given by Kane \& Gelino (2011):

$$
\begin{aligned}
\alpha & =\arccos (\cos u \cdot \sin i) \\
G(\alpha) & =10^{-0.4 \cdot\left(0.09 \cdot \frac{\alpha}{100^{\circ}}+2.39 \cdot\left(\frac{\alpha}{100^{\circ}}\right)^{2}-0.65 \cdot\left(\frac{\alpha}{100^{\circ}}\right)^{3}\right) .}
\end{aligned}
$$

Here one tries to approximate the effect that the albedo of a giant planet varies with the insolation. As one can see, the time dependence on eccentric orbits is quite complicated. Even in circular orbits, the second term is not zero in the first bracket of equation (40); therefore, this expression does not allow zero albedos. Therefore, it is allowed to the user to put slightly negative albedos as input value, but if the first bracket in equation (40) becomes negative, the code automatically sets it to zero.

It is necessary to note that the beaming effect also exhibits a time dependence of the form of $\cos u \sin i$. Therefore, I get for the expression of the out-of-transit light variation owing to beaming and reflection [approximating the $G(\alpha)$-term in the Kane-Gelino equation in the first order also by $\cos u \sin i]$ :

$$
\begin{aligned}
& B_{1}+R_{2} \approx\left(A_{2}\left(\frac{k}{a / R_{1}}\right)^{2}-\alpha_{1} \frac{K_{1}}{c}\right) \cos u \sin i \\
& B_{2}+R_{1}=\left(A_{1} f\left(\frac{k}{a / R_{1}}\right)^{2}-\alpha_{2} \frac{K_{1}}{c} \frac{n}{q}\right) \cos u \sin i .
\end{aligned}
$$

This means that the components mutually interfere between the beaming and reflection effect and show a kind of degeneracy. When albedos are measured from phase curves or from occultations, they should be corrected appropriately for beaming term. When the beaming effect is modelled in the absence of RV information, the most favourable case is $R_{2} \approx 0$. In case of strong reflection of the secondary, one cannot expect precisely measured beaming amplitudes. The measurability of this effect depends on the actual configuration.

\subsection{Correlated noise model and baseline variations}

Stars are often spotted (bright faculae and dark spots can be present beyond flares in those LCs we study). A detailed spot modelling, i.e. circular or elliptical-shaped spots changing their sizes and positions due to differential rotations, is beyond the scope of this paper and code. This is because the problem is severely degenerate, e.g. the latitudes of the spots or their diameters and temperatures can hardly be measured, owing to the fact that the overwhelming majority of the available modern, space-based, ultraprecise LCs are obtained only in white light (except a small minority of the CoRoT LCs, which have observations in three uncalibrated colours). Therefore, the temperature of the spot and hence its diameter cannot be estimated independently of each other. The exact number of spots needed to explain an LC is also a difficult question to answer.

However, it can be shown (e.g. Haywood et al. 2014) that the effect of stellar spots and faculae is summarized in the LC and acts as a random, Gaussian process. Another approach is if we assume that a spotted LC is a result of an autoregressive process, then they can appear as correlated noise in the data. Although such an approach provides parameters that can hardly be related to any physical or geometrical quantity (like the position of the spot), our purpose is not a detailed spot modelling of the stellar surface like Silva-Valio et al. (2010) do, but just to remove their impacts from the LC.

An additional factor is that we may have correlated instrumental noise in the LCs. In addition, if one bins the data - especially if they 
are the long-cadence data of Kepler - then binning may introduce red noise into the data (Ji et al. 2017).

Both effects, stellar activity and instrumental red noise, can be characterized as an autoregressive process, or as a Gaussian process or a sum of wavelets. I utilized the latter one, incorporating the routines of Carter \& Winn (2009) into the TLCM code. This is because it is faster than a Gaussian process - because the covariance matrix is known a priori - and it does not require the user to select arbitrarily defined kernels and it is not sensitive to a badly selected kernel. It contains only two free parameters to be fitted: $\sigma_{w}$ to characterize the white noise component and $\sigma_{r}$ to give an amplitude to the red-noise component. The reader should consult the paper of Carter and Winn to get more details on their wavelet-based red noise model.

When this correlated noise model is fitted, then the negative logarithm of the likelihood is minimized rather than the $\chi^{2}$ value of the fit. The routines of Carter \& Winn (2009) give directly the $\log$-likelihood of the LC fit and I denote this with $\log L$. Thus, I minimize in this case

$$
\begin{aligned}
Q= & -\log L+\frac{1}{2}\left(\chi_{\mathrm{RV}, 1}^{2}+\chi_{\mathrm{RV}, 2}^{2}\right)+\frac{1}{2} \Sigma_{k=1}^{2} \Sigma_{j=1}^{N_{\mathrm{RV}}} \ln \sigma_{\mathrm{RV}, k, j} \\
& +\frac{1}{2} N_{\mathrm{RV}} \ln 2 \pi
\end{aligned}
$$

where the terms in the brackets are the $\chi^{2} \mathrm{~s}$ of the RV curves of the primary $(k=1)$ and secondary objects $(k=2)$ and $j$ is the index of the RV observations. If any of them is not available, then they are set to be zero. The minimization of $Q$ is done in the same way as that of $\chi_{\mathrm{LC}}^{2}$. This optimization process is described in Section 4. A further explanation of equation (44) is given in Appendix D.

The corresponding LC component is denoted by $R N$ and it is the output of the Carter \& Winn (2009) routines:

$R N_{j}=R N\left(\sigma_{w}, \sigma_{r}, t_{j}\right)$.

Often, stellar spots, airmass, or instrumental effects cause a slow, parabolic-like variation in the flux. To remove this, I allow the user to fit a parabola and a user-defined function $W$ to the LC and the centre of this parabola can be anywhere in time:

$\Pi_{j}=p_{0}+p_{1}\left(t_{j}-t_{p}\right)+p_{2}\left(t_{j}-t_{\mathrm{p}}\right)^{2}+W(x)$,

where $p_{0}, p_{1}$, and $p_{2}$ are the coefficients of a parabola. $t_{\mathrm{p}}$ is the centre of this parabolic trend, which does not necessarily coincide with the epoch of transit. If $p_{1}$ and $p_{2}$ are set to zero and they cannot change but $p_{0}$ is non-zero, this means a vertical shift of the LC, which may be necessary to correct normalization or background, baseline- or contamination-subtraction problem. Sometimes even higher order baseline variations are needed, e.g. (Sedaghati et al. 2017) used a fifth-order polynomial for the correct fit. Other parameters (e.g. airmass, bias-level, FWHM of PSF, etc.) can contribute to the baseline changes. Therefore, the code allows the user to use a user-defined function $W(x)$ with fixed or free constants to model the baseline variation better. This function may have different independent variables than time $t[x=t$ is not a condition, but $x=x(t)$ should hold], e.g. $x$ can be the seeing or roll angle of a satellite, etc. The user manual gives more details on how to add such functions to the fitting procedure.

\subsection{The fitted LC model}

I add all the aforementioned effects appropriately together to get our final model. So, the model calculates the theoretically expected flux

$$
\begin{aligned}
& \left(F_{m}\right) \text { as } \\
& \begin{aligned}
F_{m}\left(t_{j}\right)= & \frac{F_{s}\left(t_{j}\right)+F_{p}\left(t_{j}\right)+l_{3}}{\left(N+l_{3}\right) \cdot(1+h)}+p_{0}+p_{1} \cdot\left(t_{j}-t_{\mathrm{p}}\right) \\
& \quad+p_{2} \cdot\left(t_{j}-t_{\mathrm{p}}\right)^{2}+W(x(t))+R N\left(t_{j}, \sigma_{w}, \sigma_{r}\right),
\end{aligned}
\end{aligned}
$$

where $l_{3}$ is the third light contribution, $h$ is a fitting parameter that is able to correct the errors in normalization, ${ }^{8}$ and $N$ is the normalization constant.

The stellar light is parametrized as

$F_{s}\left(t_{i}\right)=\operatorname{Tr}\left(1+B_{1}\left(t_{i}\right)+E_{1}\left(t_{i}\right)+R_{1}(t)\right)$,

where $\operatorname{Tr}, B_{1}, E_{1}$, and $R_{1}$ describe the light variation due to transit, ellipsoidal variation, beaming, and reflection effects for the star. ${ }^{9}$

As one can see, I consider the beaming, ellipsoidal, and reflection effects as baseline variations.

The isotropic self-emission of the planet can be measured only from secondary eclipses; otherwise, it just acts as an extra and tiny contamination factor (third light). It seems that this planetary self-emission is important only for (near-)infrared and occultation measurements, because it is smaller by orders of magnitude than the day-side radiation - reflected light - but it is comparable to the primary star in stellar binaries. Therefore, it is included in the model.

The planet's flux variation is given by

$F_{p}\left(t_{j}\right)=O c\left(n+B_{2}\left(t_{j}\right)+E_{2}\left(t_{j}\right)+R_{2}\left(t_{j}\right)\right)$.

For a planet, one can set the limb-darkening coefficients to zero, but there is an option to give non-zero values: If one models a lowmass stellar companion, then this can be necessary, or planets with atmospheres can also show limb-brightening effects.

The relationship between contamination, $c$, and third light, $l_{3}$, is

$l_{3}=\frac{c}{1-c}$,

where $l_{3}$ and $c$ are assumed to be constant in time; therefore, the change due to, e.g. a contaminating variable star should be removed by the user. $c$ tells us the fraction of the total observed light that comes from any source other than the star and the planet. Contamination may include a third, unresolved star, stray light, background galaxy, even the residuals of background subtraction, and also the effect of stellar spots and faculae. Because of faculae (bright rims in the stellar disc), $c$ can also be negative (Csizmadia et al. 2013).

The normalization constant, $N$, is taken at photometric phase $\varphi=$ 0.25 :

$N=F_{\mathrm{s}}\left(T_{0}+P / 4\right)+F_{\mathrm{p}}\left(T_{0}+P / 4\right)$.

In circular orbits, this is the moment of quadrature, i.e. we see the planet at first quarter (the same is not necessarily true for eccentric orbits!).

\footnotetext{
${ }^{8} h$ is also able to play the role of the third light. Therefore, it is not recommended to adjust simultaneously $l_{3}$ and $h$ : They are correlated to each other.

${ }^{9}$ In most of the applications, the reflected light of the planet from the star is negligible, and the same is true for the ellipsoidal variations. However, when we check yet unconfirmed planetary candidates and/or known eclipsing binaries, then we have to include these terms into the fit.
} 


\subsection{Binning and long cadence effects}

The observed light is the time integration of the above calculated flux:

$I_{m}=\frac{1}{t_{\exp }} \int_{t_{j}-t_{\exp } / 2}^{t_{j}+t_{\exp } / 2} F_{m}\left(t^{\prime}\right) \mathrm{d} t^{\prime}$.

In most of the cases, the exposure time $t_{\exp }$ is short enough that the flux can be considered constant during the exposure and then $I_{m}=$ $F_{m}$. When the exposure time is long relative to the time-scale of flux variation, the integration in equation (53) should be performed and this light value should be compared to the observed one when I calculate the goodness of the fit without red noise:

$$
\begin{aligned}
Q= & \chi^{2}=\frac{1}{N_{\mathrm{LC}}} \Sigma_{j}\left(\frac{I_{\mathrm{obs}, j}-I_{m, j}}{\sigma_{j}}\right)^{2} \\
& +\frac{1}{N_{\mathrm{RV}}} \Sigma_{j} \Sigma_{k}\left(\frac{V_{\mathrm{rad}, \mathrm{obs}, j, k}-V_{\mathrm{rad}, m, j, k}}{\sigma_{\mathrm{RV}, j, k}}\right)^{2}
\end{aligned}
$$

(for red-noise fit, see equation 44). The integration in equation (53) is carried out via a simple Simpson integration, for example:

$$
\begin{aligned}
6 I_{m} \approx & F_{m}\left(t_{j}-\frac{t_{\text {exp }}}{2}\right)+2 \cdot F_{m}\left(t_{j}-\frac{t_{\text {exp }}}{4}\right)+4 \cdot F_{m}\left(t_{j}\right) \\
& +2 \cdot F_{m}\left(t_{j}+\frac{t_{\text {exp }}}{4}\right)+F_{m}\left(t_{j}+\frac{t_{\text {exp }}}{2}\right) .
\end{aligned}
$$

The 'TimeResolution' parameter governs how many points inside the exposure time ('subexposures') are used to calculate the modelled light. The aforementioned example in equation (55) corresponds to TimeResolution $=5$, meaning that 5 points are used for the numerical integration. The user-defined TimeResolution parameter can be 1, 5, 9,17 , or 33 according to the present sets.

The usage of this kind of integration is important for the longcadence data of Kepler as well as if the bin size of the data is a significant part (about 10 percent or more) than the length of ingress/egress of transits (cf. Kipping 2010).

\subsection{Check of consistency}

When the parallax and magnitude measurements of Gaia DR2 or later data releases - and reliable - e.g. $E(B-V)$ - reddening values are available (cf. Lallement et al. 2019; Chen et al. 2019), a simple consistency check of the modelling can be carried out. The user is requested to collect Gaia magnitude $G$, parallax $\pi$, effective temperature $T_{\text {eff }}$ - an independent determination may be more precise than the Gaia value - and reddening value from some source. The mean radius of the host star is then calculated - assuming that the light contribution of the secondary is negligible - and by e.g. Monte Carlo method its error bar:

$$
\begin{aligned}
5 \log R_{\mathrm{Gaia}}= & M_{\mathrm{bol} \odot}-G-5-5 \log \pi \\
& -3.1 \cdot(1.0 \pm 0.2) E_{B-V}-B C\left(T_{\mathrm{eff}}\right)-10 \log T / \mathrm{T}_{\odot} .
\end{aligned}
$$

One may take the bolometric correction (BC) - effective temperature relationship for the Gaia photometry from Andrae et al. (2018). The factor $1.0 \pm 0.2$ reflects the fact that the interstellar absorption in the Gaia photometric band $G$ is about the same with 20 percent uncertainty than that in the $V$ band (Andrae et al. 2018).

The stellar radius can be estimated in another way by TLCM as well. Section 3 explains the details of how the Hurley, Pols \& Tout (2000) isochrones are built into TLCM, which utilizes the spectroscopic stellar metallicity, effective temperature values, and the mean density of the star measured from the fitted scaled semimajor axis to obtain the stellar radius. This stellar radius is denoted by $R_{\text {isochrone }}$ and it has an uncertainty denoted by $\sigma_{R \text {, isochrone }}$ Of course, one must have an agreement between the two kinds of the radius, so the $\chi^{2}$ or $-\log L$ values are penaltized as

$$
\chi_{\text {mod }}^{2}=\chi^{2}+\frac{1}{2}\left(\frac{R_{\text {Gaia }}-R_{\text {isochrone }}}{\sqrt{\sigma_{R, \text { Gaia }}^{2}+\sigma_{R, \text { isochrone }}^{2}}}\right)^{2}
$$

or

$$
-\log L_{\text {mod }}=-\log L+\frac{1}{2}\left(\frac{R_{\text {Gaia }}-R_{\text {isochrone }}}{\sqrt{\sigma_{R, \text { Gaia }}^{2}+\sigma_{R, \text { isochrone }}^{2}}}\right)^{2} .
$$

TLCM minimizes the modified quantities (index $\mathrm{mod}$ ).

\subsection{Usage of spectroscopic $\log g$}

When a reliable spectroscopic $\log g$ value is available, then this can be combined with the Gaia radius to get the stellar mass as

$\log M=\log g-4.43+2 \log R$,

where the logarithm has decimal basis and $g$ and the numerical factor are in c.g.s.; $R$ and $M$ are given in solar units. Therefore, the mean stellar density $\rho_{\text {star }}$ can be obtained. The mean stellar density can be also obtained from the fitted $a / R_{\text {star }}$ value (cf. equation 59 ) independently from the Gaia radius and from the spectroscopic $\log g$. Therefore, the user can use the expected $\rho_{\text {star }}$ as a prior in the fit in such cases, based on the $\log g$ value, if this option is set.

\section{ABSOLUTE DIMENSIONS}

\subsection{Method}

The transit LC fit provides relative values: the planet-to-star radius ratio, $R_{\mathrm{p}} / R_{\mathrm{s}}$, and the scaled semimajor axis, $a / R_{1}$. When one has the $\mathrm{RV}$ curves of both components (or at least the beaming effect for the two components), then one can calculate the masses of the two components via

$$
\begin{aligned}
& M_{1}=\frac{P\left(K_{1}+K_{2}\right)^{2} K_{2}\left(1-e^{2}\right)^{3 / 2}}{2 \pi \mathrm{G} \sin ^{3} i}, \\
& M_{2}=\frac{P\left(K_{1}+K_{2}\right)^{2} K_{1}\left(1-e^{2}\right)^{3 / 2}}{2 \pi \mathrm{G} \sin ^{3} i},
\end{aligned}
$$

where $\mathrm{G}$ is the gravitational constant. Using the known period value, Kepler's third law yields the semimajor axis, and then one can scale step by step the stellar radius from $a / R_{1}$ and planetary radius then from $R_{\mathrm{p}} / R_{\mathrm{S}}$ (with $R_{\mathrm{S}}=R_{1}$ ). The code calculates these automatically in this case with the corresponding error bars. Since the interstellar absorption is uncertain in many cases, the code does not calculate the $\mathrm{BC}$ and the luminosities from the radius and effective surface temperatures to get the distances.

In the case when only the primary's RV curve is measured - which is the case for exoplanets that are actually SB1 binaries - I get only the mass function:

$$
f(m)=\frac{M_{2}^{3} \sin ^{3} i}{\left(M_{1}+M_{2}\right)^{2}}=\frac{K_{1}^{3} P\left(1-e^{2}\right)^{3 / 2}}{2 \pi G} .
$$

TLCM provides two ways for the user to get the absolute masses and radii for SB1. First, it uses the effective temperature and metallicity values and substitutes them into the empirical calibrations 
of Soutworth (2011) and this gives the true mass and radius of the star. Secondly, it uses the analytic isochrone calculations of Hurley et al. (2000) and it locates the star according to its effective temperature, metallicity, and density. The density can be obtained from Kepler's third law, the measured scaled semimajor axis, and the orbital period:

$\rho_{\mathrm{s}}=\frac{3 \pi}{G P^{2}(1+q)} \frac{a}{R_{1}}$.

From the stellar isochrones, the density parameter can easily be determined at the given metallicities and metallicity uncertainties. The resolution of the data base is 0.001 in metallicity Z. TLCM checks which isochrones cross the area of the error box in $T_{\text {eff }}$ and the density parameter at the given $Z$. The average of the corresponding masses and radii in the selected isochrone parts yields the stellar mass and radius and their error bars. Then the scaling of $a / R_{1}$ and $R_{\mathrm{p}} / R_{\mathrm{S}}$ is done in the same way as before.

The density parameter provided by the code can be located by the user in any other isochrone calculation. I point out that the error budget in the planetary parameters is generally dominated by the uncertainties of the input stellar parameters. These uncertainties are discussed in Appendix A.

\section{OPTIMIZATION}

TLCM uses a genetic algorithm (GA) to find the global minimum or at least a value close to it - of the $\chi^{2}$ or $-\log L$ values. A simulated annealing (SA) algorithm is then applied to refine the fit and MCMC is used to estimate the error bars. A similar approach can be found, e.g. in Bakos et al. (2010) where an Amoeba search was utilized to find a robust solution and then they estimated the uncertainties via MCMC. A different version of SA was successfully applied to solve and refine eclipsing binary LC parameters in, e.g. Prša \& Zwitter (2005).

\subsection{Optimization by GA}

The GA is based on the so-called 'Harmony Search' realization (HS), which is a robust, fast, and reliable method to find the global minimum (Geem, Kim \& Lonatathan 2001). Of course, no method is guaranteed to find the global minimum in every case, but Gao et al. (2015) illustrate using many examples that HS provides fast and good performance. I tested extensively the method on 2000 synthetic LCs with different signal-to-noise ratios and we found very good results (for details, see Csizmadia et al. 2013). HS maps the whole range of a prescribed parameter space quite effectively as I show hereafter.

The interested reader can find examples in Charbonneau (1995), Metcalfe (1999), and Attia et al. (2009) to see how well GAs perform for binary stars whose LC modelling is a very similar problem to planetary transit fits.

Hereafter, bold-faced letters mean $N_{\mathrm{p}}$ element vectors; $N_{\mathrm{p}}$ is the number of free parameters. I added constraints (box priors in Bayesian terms) to HS; thus, I search for the solution around $\mathbf{p}_{0}$ within $\mathbf{B}_{0}$. Here $\mathbf{p}_{0}$ is the centre of the searching box and $\mathbf{B}_{\mathbf{0}}$ is its half-size, both are vectors. E.g. $\mathbf{p}_{0}=\left(a / R s=10, k=0.1, b^{\prime}=0.5\right)$ and $\mathbf{B}_{0}=(9,0.1,0.5)$. This means that a solution is searched in the parameter range (1...19) for $a / R s,(0 \ldots 0.2)$ for the radius ratio, and (0...1) for the conjunction parameter and all solutions outside these limits are ignored.

Let us assume for a moment that our fit has three free parameters: $a / R_{\mathrm{s}}, k$, and $b^{\prime}$. Then the vector $\mathbf{p}_{1}=(10,0.1,0.5)$ is an individual with $\left(a / R_{\mathrm{s}}\right)_{1}=10, k_{1}=0.1$, and $b_{1}^{\prime}=0.5$. The elements of this vector are called genes, e.g. $p_{11}$ is a gene corresponding to $p_{11}=a / R s=$
10 in this example. TLCM creates $N_{\text {init }}$ such vectors and the group of all individuals is called the population. The only constraint is that $2<N_{\text {init }}<2000$. Usually, $N_{\text {init }}$ is between 25 and 1000. Although good results were found with $N_{\text {init }}=25$ for many synthetic LCs, I recommend to use at least $N_{\text {ninit }}=100$ to avoid converging too quickly and finding a local minimum instead of the global one. ${ }^{10}$

Every individual is randomly selected from the searching box (except the first individual that takes the values of the centre of the searching box). Every individual has a fitness parameter, which can be either $\chi_{i}^{2}$ or $-\log L_{i}$ and the fitness is better if its value is smaller in this problem.

Then the HS, as adapted by me, consists of the following steps:

(1) Create 2000 random sets of parameters and calculate the corresponding fitness values. Then select the smallest $N_{\text {init }}$ fitness values. This is a Monte Carlo-like search for the solutions, which is not very effective after a while; therefore, it stops at 2000 trials. Note that Step 1 is not part of the standard HS. It gives the advantage of a better starting position for the subsequent steps. Since it takes no significant CPU time, it is recommended to apply this step.

(2) I start an iteration with the iteration number $l$. Select uniformly distributed random numbers $R_{1,2,3,4,5}$, each of which is between $0 \ldots 1$ and which are independent of each other and their values are changed in every iterational step. Some of these quantities are vector random numbers, whose elements are random numbers.

(a) If $R_{1}>0.999$, then $\mathbf{p}_{\text {test }}=\mathbf{p}_{\mathbf{0}}+2 \cdot\left(\mathbf{R}_{\mathbf{2}}-0.5\right) \cdot \mathbf{B}$. This step is just an absolutely random search (a Monte Carlo step) in the whole investigated parameter space, which helps to keep the predictive power of the algorithm. ( $\mathbf{R}_{\mathbf{2}}$ is a vector of $N_{\mathrm{p}}$ different random numbers.)

(b) If $R_{1}<0.999$, then the new test-individual $t$ is built up from $N_{\mathrm{p}}$ different individuals: If $R_{3}<0.999$ (the majority of cases), then $t_{n}=p_{k n}$, where $k$ is a random integer number between $1 \ldots N_{\text {init }}$ and if $R_{3} \geq 0.999$ then $p_{\text {test }, n}=p_{0 n}+2 \cdot\left(R_{4}\right.$ $-0.5) \cdot B_{n} . n$ is the index of the gene. This means that every gene originates from a different chromosome (Fig. 7) and this is the main difference relative to the Asexual Genetic Algorithm (Cantó, Curiel \& Martínez-Gómez 2009) where every new child comes from only one parent and to Charbonneau (1995) traditional GA where every child has only two parents. In HS, a child has as many parents as genes. The derivation of new individuals means that $\mathrm{HS}$ is able to create children that are very far from the parents (the apple may fall very far from its tree in HS) and therefore it is able to map regions of the searching box that are quite far from the nearby location of the parent individuals.

(c) The next step is mutation. The mutation rate is governed by a random number $M$ that lies in the range $0 . .1$, and its actual value is valid for every $N_{\text {init }}$ steps; after that I choose another random $M . \mathbf{R}_{5,6}$ are vectors with $N_{\mathrm{p}}$ independent random numbers. $\mathbf{R}_{5}$ 's components are uniformly distributed random numbers between $0 . . .1$, while $\mathbf{R}_{6}$ 's components follow a normal distribution with zero mean and unit standard deviation. If $R_{5 s}<M$ for the $s$ th gene, then $t_{j s}=t_{j s}+R_{6 s} * S\left(p_{\text {all, } s}\right)$. Here $S\left(p_{\mathrm{all}, s}\right)$ means the standard deviation of the $s$ th components of all $p$ s. As the algorithm converges, the standard deviation of the parameters will be smaller and smaller, so the mutations will

\footnotetext{
${ }^{10}$ See also the footnote of Eastman et al. (2013) about the length of pseudorandom sequences, which may have an effect on the solutions.
} 


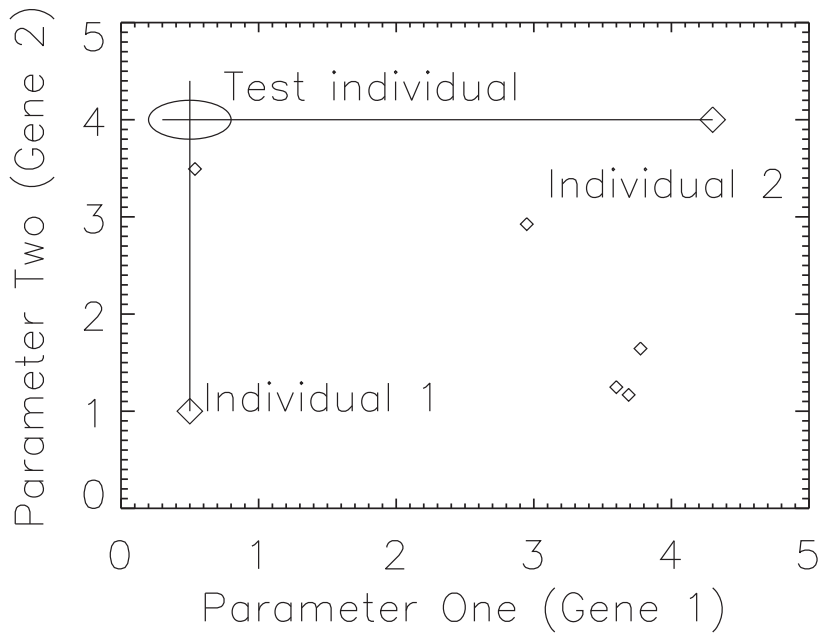

Figure 7. An illustration of how Harmony Search selects trial solutions (test individuals) in 2D. There are several individuals (denoted by diamonds), and their group is called the population. Two of them are randomly selected (denoted by larger diamonds, and called individuals 1 and 2). The first randomly selected individual lends its first gene (=parameter 1) and the second randomly chosen individual lends its second gene (=parameter 2). This defines a central point around which a small ellipse is drawn according to some prescribed value (e.g. the standard deviation of the population). A small mutation is added, which has a Gaussian distribution with scatter equal to the axis sizes of the ellipse, and the corresponding $\chi^{2}$ value of this trial solution is calculated. If it is better than the worst solution in the population, then the worst is replaced by this trial solution. From time to time, with small probability, I try totally random trial solutions or one of the genes is selected randomly from the whole parameter range under consideration.

be also smaller. This self-governing property of the algorithm provides convergence.

This kind of mutation maintains genetic variety even when $M$ is by chance very small. Note that during the whole iteration process the expected value of $M$ will be 0.5 .

The code does a check not to leave the searching box; if that happens I try another random $\mathbf{R}_{\mathbf{2}}, R_{3}$ numbers.

(d) Then the corresponding fitness value is calculated. If this is smaller than the one with the highest fitness in the actual population, then I replace this worst individual with $\mathbf{t}_{l}$.

(e) Steps (a)-(d) are repeated until one of the following two criteria is fulfilled: (i) the ratio of the maximum and minimum $\chi^{2}$ values in the current population falls below 1.001; (ii) the maximum number of iterations is reached. This is set to $300 \times$ $N_{\text {init }}$ (in other words the maximum number of generations is 300). In practice, I experienced that about 40-60 generations are enough in many cases to reach the first terminating criterion.

Usually the GA part is computationally inexpensive. Compared to other methods, it is much faster and more reliable as a means of finding the global minimum than other methods like AMOEBA, LM, ant colony, and firefly - see the compilation of examples by Geem (2009).

\subsection{Refinement by SA}

The HS solution is defined as an average of the final generation. This solution is refined by SA. SA is an algorithm to optimize a function and actually it is a method quite close to the well-known Monte Carlo Markov Chain processes. I do not need to map the whole parameter space again; I want to explore only the small vicinity of the GA solution. Therefore, I implemented SA in the following way.

One SA chain starts with the parameter values found by the GA procedure: $\mathbf{t}_{\text {old }}=\mathbf{t}_{\text {final, GA }}$. Then a new test-parameter set is produced by a random perturbation of the previous state with

$\mathbf{t}_{i}=\mathbf{t}_{\text {old }}+\mathbf{w} \cdot \mathbf{r}_{i} \cdot \mathbf{v}$,

where $\mathbf{w}$ is a $N_{\mathrm{p}}$-element vector containing $0 \mathrm{~s}$ and $1 \mathrm{~s}$. These two numbers are chosen randomly for every parameter with the rules that at least one 1 should be in the $\mathbf{w}$ vector and the fraction of $1 \mathrm{~s}$ is equal to or less than $0.25+0.75 / N_{\mathrm{p}}$. The constants 0.25 and 0.75 are heuristic ones. The goal of this multiplicative factor is to imitate the Multiple Subset Method (e.g. Wilson \& Biermann 1976), so it keeps some of the parameters constant and others are free during one step; in the next iteration step another $\mathbf{w}$ is chosen, also randomly. This helps to resolve the possible degeneracies and intercorrelations between the parameters. Since I do not know a priori which parameters are strongly correlated, I select different subsets every step.

The code calculates the quantity

$P_{i}=\exp \left(-\left(\chi_{i}^{2}-\chi_{\mathrm{old}}^{2}\right) / 2 T_{\mathrm{SA}}\right)$.

$T_{\mathrm{SA}}$ is the so-called 'SA temperature' that is unrelated to any physical temperature of the modelled objects. $\mathbf{t}_{\text {old }}$ and $\chi_{\text {old }}^{2}$ are replaced by $\mathbf{t}_{i}$ and $\chi_{i}^{2}$, respectively, if $P_{i}>u$, where $u$ is a uniformly distributed random number between $0 \ldots 1$. Note that that $P_{i}>1$ always when $\chi_{i}^{2}<\chi_{\text {old }}^{2}$.

The number of accepted solutions relative to all trials is the acceptance rate. Solutions with better fitness are always accepted.

$T_{\mathrm{SA}}$ is adjusted until I have an acceptance rate of 24 per cent (with \pm 1 per cent tolerance), which is a recommended value for MCMC in multiparameter fits (e.g. Dunkley et al. 2005). When this is reached, SA is restarted with this well-adjusted $T_{\mathrm{SA}}$.

The stepsize $\mathbf{v}$ is initially defined by the standard deviation of the parameters in the final GA generation, but after setting the SA-control parameter $T_{\mathrm{SA}}$, it is updated every iteration step: If the acceptance rate of the iterational steps falls below 23 per cent, TLCM decreases the stepsize little bit, and if it is over 25 percent, the stepsize is increased.

SA is almost identical to MCMC but it has one main difference to MCMC. SA differs from MCMC in the sense that I change $T_{\mathrm{SA}}$ after every $N=2000$ iteration steps: $^{11}$

$T_{\mathrm{SA}} \rightarrow T_{\text {change }} \cdot T_{\mathrm{SA}}$,

where $T_{\text {change }}$ is a user-defined number. The decrease of the 'SA temperature' ensures that I do not leave the found - likely global - minimum region and it will be mapped effectively (the apple does not fall far from its tree now). Note that the hyperspace of the LC modelling has a very complicated structure (Milone \& Kallrath 2009). The Markov Chain processes are less effective because they need a lot of iteration steps and thus CPU time, and their performance depends on the probability distribution of the parameters, which is not known a priori (Dunkley et al. 2005).

MCMC is able to 'go up the hill', which means that it accepts worse solutions sometimes and in this way it escapes from the found minimum even if it is a global minimum. Although it is able to go back to the global minimum, it does this in a random way and generally after many steps. It is not needed that MCMC explores the parameter range because it was done more efficiently already in the GA phase of the optimization. One needs to explore the vicinity of

${ }^{11}$ The choice of 2000 is arbitrary. 
the GA-found minimum. Therefore, I use SA to force the Markov chains not to go far from the solution GA found. The decrease of the 'SA temperature' keeps the solution close to the found likely global minimum range, saving CPU time but keeping the reliability of the solution too.

Note that when one sets $T_{\text {change }}=1$ and all components of vector $\mathbf{w}$ are 1 , then the method is equivalent to MCMC. When $T_{\text {change }}=0$ only better solutions are accepted, and when it is much bigger than unity then it becomes a non-controlled random walk.

\subsection{Uncertainty of the parameters}

The uncertainties of the derived parameters are estimated by running two or more MCMC chains. Now the 'SA temperature' is not changed and the $\mathbf{w}$ vector contains only $1 \mathrm{~s}$ in equation (60). The adjustment of the stepsize is done as it was done for SA. The first 6000 steps are discarded automatically from the chain. The convergence tests described in Section 4.4 should be watched by the user to see if convergence is reached or a longer chain is needed, and the user must check whether the overall acceptance rate was close to 23 per cent or not.

TLCM uses the recommendation of Dunkley et al. (2005) to run shorter MCMC chains, allowing one parameter only to vary and adjusting the width of the proposal distribution until 90 percent of acceptance rate is reached in every of these chains. Then another shorter chains were run when all free parameters could vary and the proposal distribution have been multiplied with the same factor until 23 per cent acceptance rate was reached. Once the right stepsizes were found, the MCMC is restarted with the prescribed number of steps and the stepsizes are kept constant.

The lower and upper $1 \sigma$ error bars are estimated via a common 16-84 per cent rule, and the final solution is the median of the MCMC chains.

The code is also able to perform a bootstrap analysis for estimating the uncertainties. However, it takes more CPU time than MCMC and usually provides smaller error bars. This can be an inherent property of bootstrap.

\subsection{Convergence test}

TLCM runs at least two different SA and MCMC chains, but the user can define a higher number of chains. The first chain starts from the best solution GA found; the starting points of the others are taken from randomly selected solutions around the best one. Usually, SA refines further a little bit the GA solution. For each free parameter separately the standard deviation of the parameter in the whole sample (denoted by $W$ ) is calculated, and the standard deviations of the mean solutions of every chain (denoted by B) are determined. Then I calculate the Gelman-Rubin (e.g. Croll 2006, and references therein) convergence number for every parameter:

$R=\frac{n_{l}-1}{n_{l}}+\left(1+\frac{1}{n_{\mathrm{c}}}\right) \frac{B}{W}$.

Here $n_{l}$ is the chain length and $n_{\mathrm{c}}$ is the number of chains. When this $\mathrm{R}$ is over 1.1 for any of the parameters, then there is a degeneracy in some of the parameters (the chains converged to different values) or the chain was too short and convergence was not reached (Croll 2006). Then it is advised to repeat the analysis with longer chains or with different settings.

I used another heuristic convergence test too. Since SA, MCMC, GA, and several other methods depend on the random numbers used for the iteration process, one cannot expect that the results will be the same after each new start (except if one uses the same random number sequence for the new run, which is a mistake). However, one can expect, suggested by the theorem of big numbers, that the solutions will be reasonably close to each other if a code is rerun twice or more. The 'reasonably close' is a matter of convention. Let us define the quantity $X$ to measure the deviation of two runs for a certain parameter $p$ as

$X=\frac{p_{1}-p_{2}}{\sqrt{\sigma_{1}^{2}+\sigma_{2}^{2}}}$

and this is calculated for each parameter separately. It is assumed for this analysis that the distributions of the likelihoods in the two runs are normally close to the solution. Here, $p_{i}$ and $\sigma_{i}$ are the peak of this distribution and its scatter.

The cumulative probability distribution function (CDF) gives the probability that this $X$ can be smaller than some $X_{\text {c }}$ cut-off value just by chance. The CDF of Student's $t$-distribution will give us an answer that these two solutions differ from each other just by chance by the value $X$. The choice of the cut-off value is somewhat arbitrary; for this work $X_{\mathrm{c}}=0.1$ was selected. That means that we calculate the probability that two code runs produce smaller difference in the end results than 10 per cent of the quadratically weighted average of their $1 \sigma$ error bars. Note that statistically speaking even $1 \sigma$ or $3 \sigma$ difference could not be stated as 'statistically significant' difference, so taking this limit we have a strict criterion to get the same answer after two runs. Recall that CDF gives the probability

$\mathrm{CDF}=P\left(X<X_{\mathrm{c}}\right)$.

We expect that $X$ will scatter around zero; the probability that it differs from zero not better than $\pm X_{\mathrm{c}}$ is given by

$P\left(-X_{\mathrm{c}}<X<+X_{\mathrm{c}}\right)=P\left(X<+X_{\mathrm{c}}\right)-P\left(X<-X_{\mathrm{c}}\right)$.

Using the expression available in textbooks for Student's $t$ distribution, I have got that

$P(-0.1<X<0.1)=0.472 ;$

therefore, rearranging equation (64), the maximum allowable difference between the runs is

$\Delta p_{\text {allowable }}=0.472 \sqrt{\sigma_{1}^{2}+\sigma_{2}^{2}} \approx 0.472 \bar{\sigma}$,

where $\bar{\sigma}$ is the quadratic average of the error bars. Similar argumentation can be taken when one compares the results of two studies or two codes.

An example is shown for such a convergence test in Fig. 8 and in Table 3. I used the CoRoT LC of CoRoT-20b's transits and performed fits with different chain numbers and chain lengths for the SA part. I repeated the experiments with the different settings 20 times. In Table 3 the maximum difference of the derived parameters in the 20 runs is tabulated.

The total number of iterations was often the same (200 000), but in the first two attempts 2 chains $\times 100000$ steps/chain were carried out with different SA temperatures. Two other experiments consisted of 50 chains $\times$ with 4000 and 10000 steps/chain, and the last one was based on 400 chains $\times 500$ steps/chain.

The conclusions drawn from Fig. 8 and Table 3 are as follows:

(i) The maximum allowable differences in the main parameters are as follows: 0.65 (in $a / R_{\mathrm{s}}$ ), 0.0017 (in $R_{\mathrm{p}} / R_{\mathrm{S}}$ ), 0.09 (in conjunction parameter $b^{\prime}$ ), and 0.07 and 0.23 in $u_{+}$and in $u_{-}$, respectively.

(ii) Using only two chains with 100000 steps, the difference between the separate runs is greater than the aforementioned prescribed limits, although decreasing the SA temperature gives slightly better results and in $k$ the condition given by equation (68) is already 

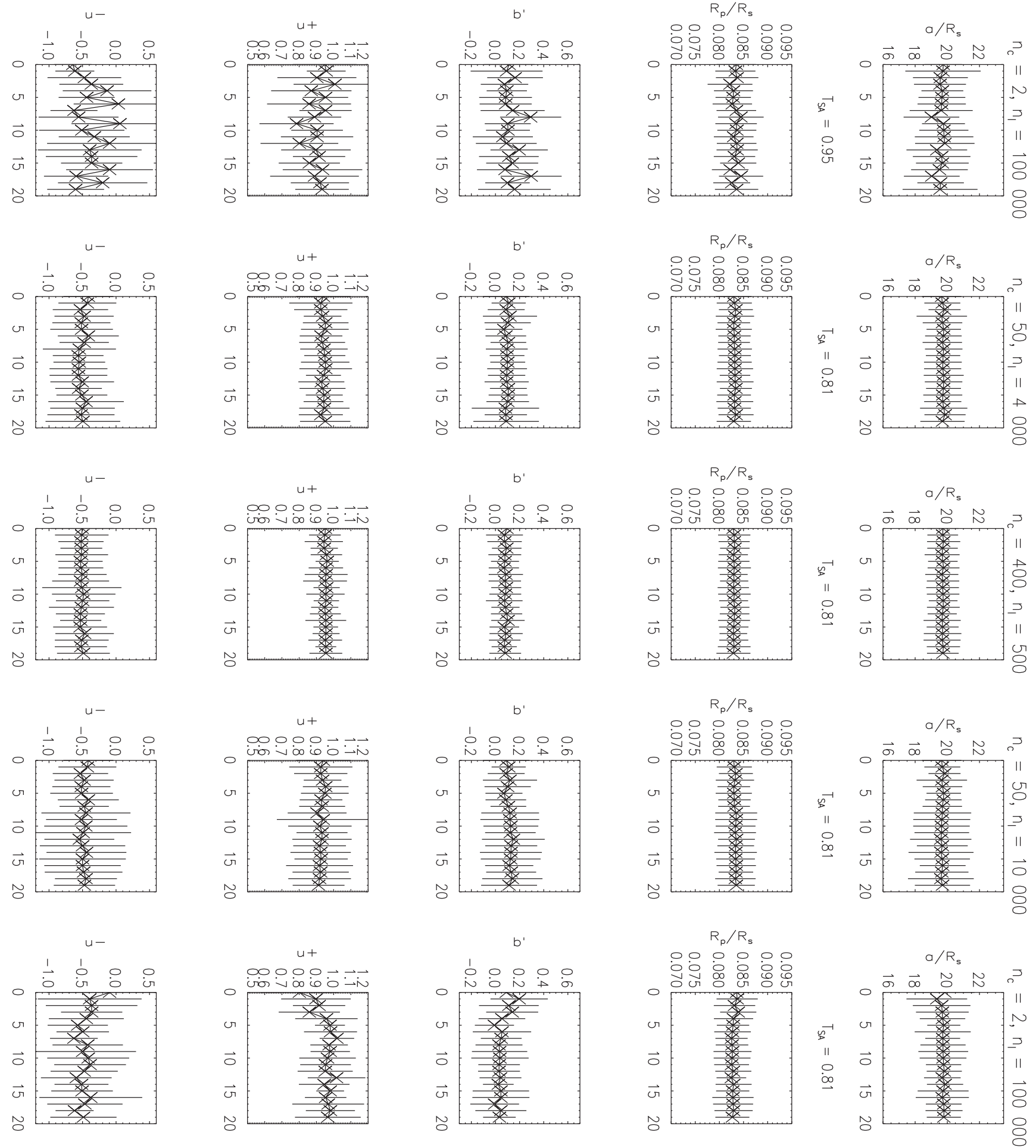

Figure 8. Convergence test results. The columns represent one convergence experiment with different settings for the SA run, and horizontally one can see the different parameters whose convergence were investigated $\left(a / R_{\mathrm{S}}, k=R_{\mathrm{p}} / R_{\mathrm{S}}, b^{\prime}, u_{+}, u_{-}\right)$. Each cross gives the end result of one run and the vertical lines are the corresponding error bars. The $x$-axis represents the run number. The $y$-axis scales are always the same within one row for sake of easier comparison. When the number of chains is only two (first and last columns), there are fluctuations in the end results from one run to another one, especially in the limb darkening parameters and in the conjunction parameter. When the number of chains is 50, the solutions starts to stabilize and the fluctuations are much smaller among the consecutive runs. When the number of chains is 400 , stable solutions are obtained. 
Table 3. Comparison of different chain numbers, chain lengths, and SA temperatures. $\max (p)-\min (p)$ means the maximum and minimum values of a parameter in the tried 20 runs.

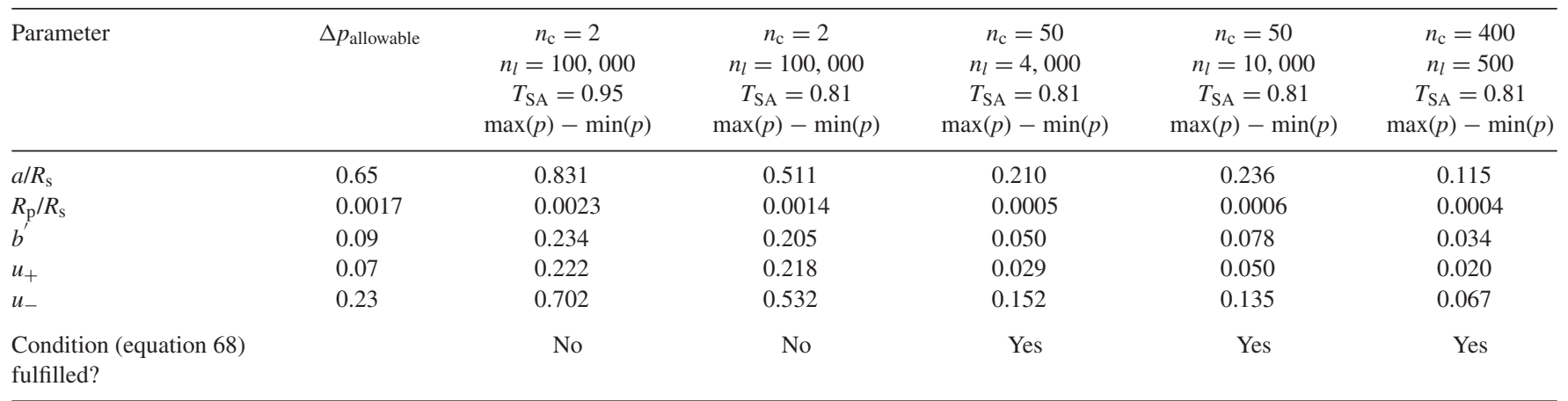

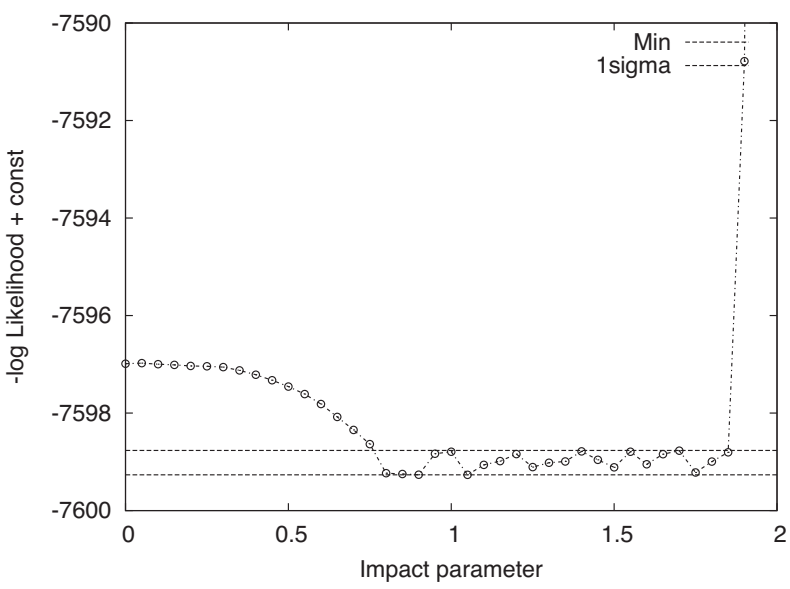

Figure 9. Result of a grazing transit test. The shifted minus log-likelihood is plotted against the fixed impact parameter. Scaled semimajor axis, radius ratio, red noise parameters, etc. were fitted to NGTS-1b (Bayliss et al. 2018). The minimum of the found $-\log L$ values and the $1 \sigma$ uncertainty ranges are plotted. As one can see, the fit is better if the impact parameter changes from zero to about 0.8 . After that, the fit quality is practically constant. The very last point has a worse fitness parameter because the radius ratio was constrained and the fit converged to the side of the searching box. From this figure, the grazing nature is clear and one can say only that the impact parameter is bigger than 0.8 but an upper limit cannot be given.

fulfilled. I also tried smaller values for $T_{\text {change }}$ and it seems that it provides very nice results. However, complicated LCs of some selected Kepler eclipsing binaries produced better results with two longer chains (up to 100000 ) and with high control parameter for SA ( $T_{\text {change }}=0.98$ ). Determining the optimal value of $T_{\mathrm{SA}}$ will be the subject of another study.

(iii) Each of the other three combinations of chain numbers and chain lengths resulted in deviations well within the prescribed limits. The smallest deviations and therefore best results were obtained by 400 short chains (chain lengths were 500 only), but 50 chains (4000 and 10000 steps in each chain) also produced acceptable results.

Therefore, it seems that two chains can perform quite close to the desired limits, but one has to recommend using more chains to get a stable and good result. The chain length can be as small as 4000, but these recommendations are based only on this particular case. It will be the subject of another study to explore how this stability and convergence depends on, e.g. the signal-to-noise ratio of the data.

Fig. 4 of Csizmadia et al. (2013) shows another experiment with the code. 2000 synthetic LCs with different transit depths/white photometric noises ( $d / \sigma$ ratio) were modelled and it was found that the code is able to recover the parameters very well even for noisy LCs. According to that test, if the conjunction parameter is less than 0.85 , then $d / \sigma>25$ is needed to recover the semimajor axis better than 5 percent, $d / \sigma>50$ for recovering radius ratio better than 1 percent, $d / \sigma>100$ to recover $b^{\prime}$ better than 10 percent and the same limit is valid to recover the limb-darkening coefficients better than 3 percent. It was also found that if $b^{\prime}>0.85$ then the solution for shallow transits becomes uncertain because we lose the inner contacts and the corresponding information.

Beyond the Gelman-Rubin statistics and the heuristic test presented here, other useful convergence tests are mentioned, e.g. in Ford (2006). When the user wishes, these tests can be carried out because the chains are saved into a separate file.

\subsection{Parameter and uncertainty checks}

The code checks the input values and non-physical values. In the case of period, scaled semimajor axis, radius ratio, third light, albedos, mass ratio, effective temperature, and surface brightness ratio, only positive values are accepted. In the case of $\sqrt{e} \sin \omega$ and $\sqrt{e} \cos \omega$, if the eccentricity becomes larger than 0.9999 , then it is set automatically to 0.9999 as the maximum allowed eccentricity in TLCM. The impact of the parameter checks on the posterior distributions is not studied so far.

If any of the proposed parameter value is beyond the prescribed limits of the searching box, the code replaces this proposal by a new one until the proposal will fall inside the prescribed searching box.

The user is asked to check the solutions after the code's run finishes, and if they converge to the edge of the searching box, then probably the searching box size was too small; hence, its location and/or size should be changed. It is also a good practice if the posteriori distribution peak of the MCMC chains is close to the centre of the searching box; otherwise, significant biases can occur in the estimate of the median and $1 \sigma$ limits.

\section{GRAZING TRANSITS}

Grazing transits are transits where the condition

$1-k<b^{\prime}<1+k$

holds; in other words, the full planetary disc does not cover the stellar disc. In such cases where the inner contacts are missing, there is no so-called 'flat part' of the transit and the information content is much less. More severely, the impact parameter cannot be fitted. An example is shown in Fig. 9 showing the $-\log L$ as a function of fixed 
Table 4. Results of joint LC-RV curve modelling of CoRoT-1b and comparison to the results of Barge et al. (2008). TLCM used NINIT $=1000, \mathrm{nmax}=100000$, and nchain $=10$ with a thinning factor of 10. For TLCM modelling, the same effective temperature and metallicity were applied as in Barge et al. (2008). The last column gives the differences between the two solutions in terms of 'sigmas'. This is defined as: $|a-b| / \sqrt{\sigma_{a}^{2}+\sigma_{b}^{2}}$, where $a$ and $b$ are the same parameters from the two solutions and $\sigma_{a, b}$ are the two error bars in the two studies.

\begin{tabular}{lccc}
\hline Parameter & Barge et al. (2008) & TLCM & \\
\hline$V_{\gamma}\left[\mathrm{km} \mathrm{s}^{-1}\right]$ & $23.354 \pm 0.008$ & $23.326 \pm 0.021$ & $1.2 \sigma$ \\
$K\left[\mathrm{~m} \mathrm{~s}^{-1}\right]$ & $188 \pm 11$ & $188 \pm 17$ & $0 \sigma$ \\
$R V_{\text {drift }}\left[\mathrm{m} \mathrm{s}^{-1} \mathrm{~d}^{-1}\right]$ & $\sim 1$ & $1.03 \pm 0.1$ & - \\
$T_{\text {eff }}[\mathrm{K}]$ & $5950 \pm 150$ & - & - \\
{$[M / H]$} & $-0.3 \pm 0.25$ & - & - \\
$M_{\text {star }}$ & $0.95 \pm 0.15$ & $0.95 \pm 0.16$ & $0 \sigma$ \\
$R_{\text {star }}$ & $1.11 \pm 0.05$ & $1.12 \pm 0.06$ & $0.1 \sigma$ \\
$M_{\text {planet }} / M_{\text {Jup }}$ & $1.03 \pm 0.12$ & $1.03 \pm 0.14$ & $0 \sigma$ \\
$R_{\text {planet }} / R_{\text {Jup }}$ & $1.49 \pm 0.08$ & $1.53 \pm 0.10$ & $0.3 \sigma$ \\
Epoch -2450000 & $4159.4532 \pm 0.0001 \mathrm{JD}$ & $4159.45296 \pm 0.00008 \mathrm{HJD}$ & $1.8 \sigma$ \\
$u_{+}$ & $0.71 \pm 0.16$ & $0.60 \pm 0.11$ & $0.6 \sigma$ \\
$u_{-}$ & $0.13 \pm 0.30$ & $0.10 \pm 0.31$ & $0.1 \sigma$ \\
$a / R_{\text {star }}$ & $4.92 \pm 0.08$ & $4.86 \pm 0.06$ & $0.6 \sigma$ \\
$R_{\text {planet }} / R_{\text {star }}$ & $0.1388 \pm 0.0021$ & $0.1403 \pm 0.0014$ & $0.6 \sigma$ \\
$i$ [deg $]$ & $85.1 \pm 0.5$ & $84.8 \pm 0.4$ & $0.5 \sigma$ \\
$p_{0}[\mathrm{ppm}]$ & - & $-27 \pm 20$ & - \\
\hline & & &
\end{tabular}
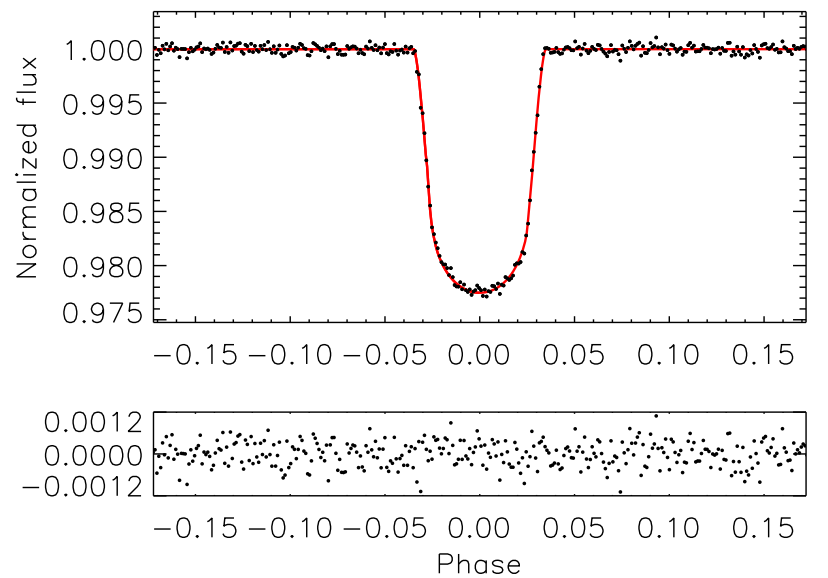

Figure 10. The LC of CoRoT-1b. Upper panel: the black points are the observations; the red solid curve is the LC fit. Lower panel: the residuals of the fit.

impact parameter values for NGTS-1b (Bayliss et al. 2018). As one can see in such a grazing case, only a lower limit can be given for the impact parameter.

Since the transit duration is linked to the impact parameter in circular orbits as

$D=\frac{P}{\pi} \frac{R_{\mathrm{s}}}{a} \sqrt{(1+k)^{2}-b^{2}}$,

there is a mutual degeneracy between the scaled semimajor axis $a / R_{\mathrm{s}}$, the radius ratio $k$, and the impact parameter $b .{ }^{12}$ The analysis of this equation shows that if we increase $b$, then its change is fully

\footnotetext{
${ }^{12}$ Equation (70) can be obtained from equation (4) by substituting $e=0$, $\delta=1+k$, which is valid in the moment of the first and last contacts, and approximating $\sin ^{2} i \approx 1$ as well as $\cos \left(v_{1}+\omega\right) \approx 1-(D / P)^{2}$ in the moment of the first contact.
}

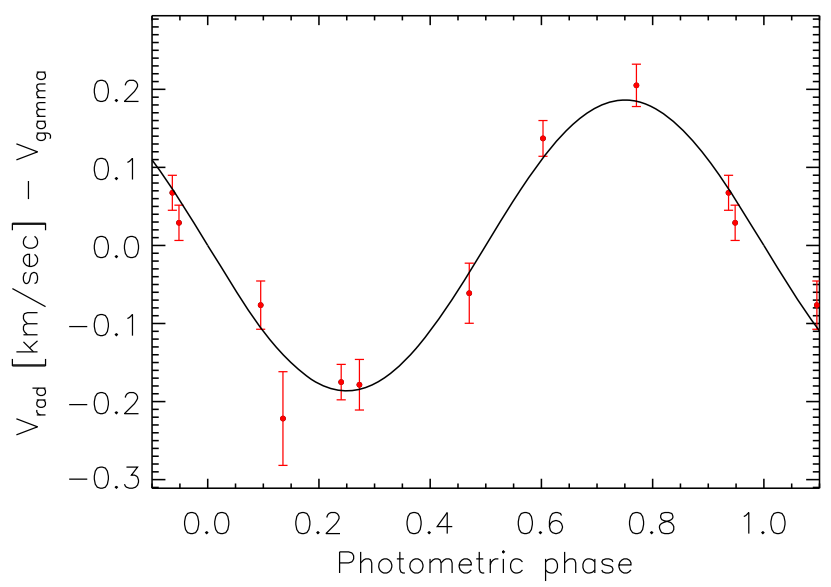

Figure 11. The RV curve of CoRoT-1b. The red points show the observed $\mathrm{RV}$ data together with their uncertainties and black curve is the model fit by TLCM. The observed data points were corrected for the RV drift phenomenon (Table 4, Section 8.1).

compensated by increase of $k$, so one can get only a lower limit for the radius ratio in such cases. It is important to emphasize that in case of grazing transit the transit depth does not give the value of the radius ratio because only part of the planetary disc plays a role in the transit (again, only a lower limit can be given).

TLCM offers the possibility that it fixes the conjunction parameter between certain user-defined limits (e.g. between 0 and 2 with $\Delta$ $b^{\prime}=0.05$ resolution like in Fig. 9) and it searches for solutions at fixed impact parameters. Then the observed $Q$ values can be used to estimate the lower limit of conjunction parameter and radius ratio (cf. Fig. 9).

In Csizmadia et al. (2015), it was shown that if one has the stellar density (e.g. from asteroseismology, or isochrone-fitting to spectroscopic parameters and applying Kepler's third law), the value of $a / R_{\mathrm{S}}$ can be constrained and it can be fixed or it can vary around 


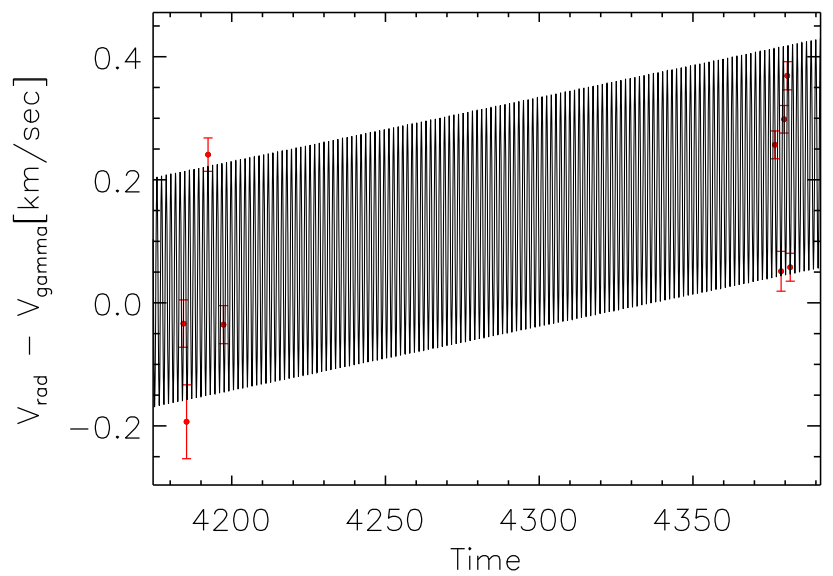

Figure 12. RV drift in CoRoT-1b. The black curve is the model fit and the red points with vertical lines are the observed data and their uncertainties.
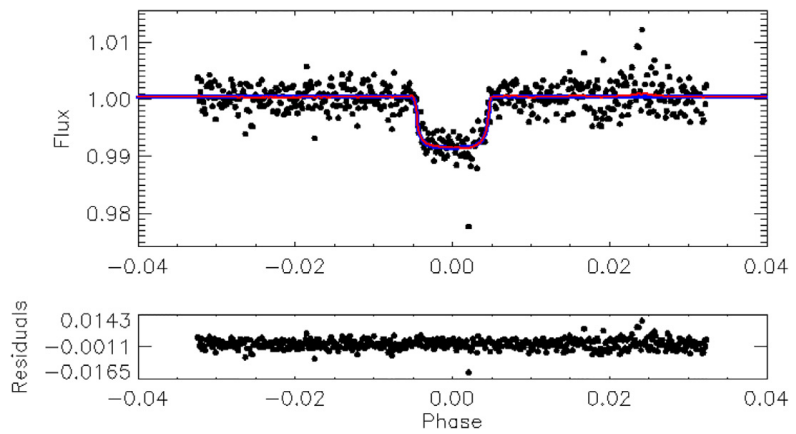

Figure 13. LC solution of CoRoT-20b with TLCM. Upper panel: the black dots show the processed, normalized observed fluxes of CoRoT-20. The transit of the planet $b$ is clearly visible. The blue curve is the fit without the red-noise effects. The red curve shows the transit fit plus the red-noise effects. The red curve is the optimized curve during the fit procedure. Lower panel: the residuals after subtracting the transit model + red noise model. The red noise is not significant in this LC.

a well-defined value within its uncertainty ranges. This resolves the degeneracies due to the grazing transit nature.

\section{MULTICOLOUR LCS AND STELLAR SPOTS}

The released version of TLCM analyses only single-colour LCs, although an ad hoc version already exists for four channels. A version was produced for analysis of multicolour LCs with arbitrary number of channels (MSc thesis of Sándor Kunsági-Máté, Eötvös University Budapest, 2018). Including an analytic stellar spot model is also a feature I would like to introduce in the future.

\section{AVAILABILITY OF THE CODE}

The code is running under IDL (version 6.1 or higher), and can be used in batch mode. The code was tested under the free GDL and it runs under it (Windows, Unix, or Linux).

The code and its user manual are freely available and can be downloaded from the following homepage: www.transits.hu.

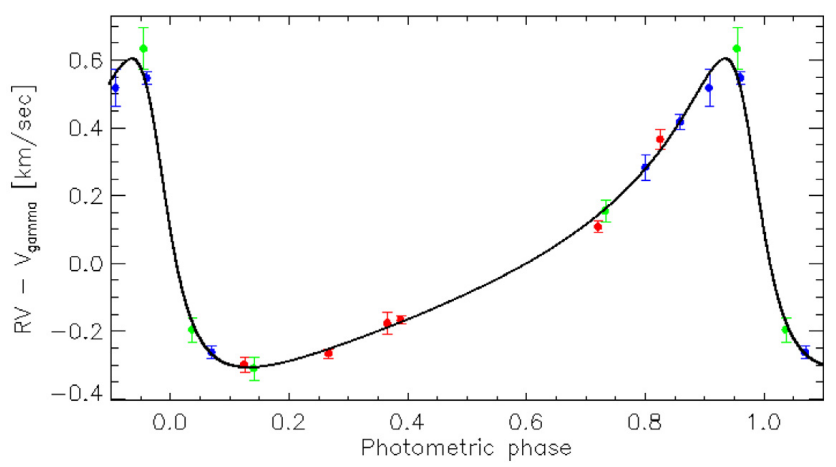

Figure 14. RV curve of CoRoT-20b and its fit obtained by TLCM. Different colours represent RV data obtained by different instruments: green: FIES, blue: HARPS, and red: SOPHIE. Details of the observations can be found in Deleuil et al. (2012).

\section{EXAMPLES AND VALIDATION}

Although studies where TLCM was used were listed in the 'Introduction' section, several new features - e.g. the out-of-transit features, red-noise fit - were added to the code in the meantime. In order to show and validate the new capabilities of the code, the following results are presented hereafter.

\subsection{CoRoT-1b}

CoRoT-1b was the first exoplanet discovered from space (Barge et al. 2008). The RV data were taken from Barge et al. (2008) but I converted the time records given in JD therein to HJD, because the CoRoT photometric data are already in HJD. The photometric data were obtained from the public CoRoT archive and they were reduced in the same way as in the case of CoroT-20b (see Section 8.2).

A fixed eccentricity was used. The fitted parameters and the results are summarized in Table 4 where the reader can find the results of Barge et al. (2008) for comparison. Notice that Bonomo et al. (2017) confirmed the existence of the RV drift, too.

The results are shown graphically in Figs 10-12. The observed RV drift in Fig. 12 shows that this system deserves a new RV study to confirm the presence of the drift and to establish its exact nature and cause.

The agreement between the results obtained by Barge et al. (2008) and provided by TLCM is good and this validates the code. The error bars in the stellar mass are, however, smaller than those in Barge et al. (2008), maybe due to different isochrones being used. This is also reflected in the smaller error bars in the planetary mass. The other error bars are very close to the ones in Barge et al. (2008), sometimes slightly bigger (like in the case of $K$ and $V_{\gamma}$ ) or smaller than those in their cases, but the difference is not significant.

\subsection{Modelling of CoRoT-20b}

Transits of CoRoT-20b were detected by Deleuil et al. (2012). Although the present author was co-author of that paper, the joint transit LC and RV modelling was done by a different code: Formalism of Gimenez (2006a) and AMOEBA-optimization was utilized as explained there. Therefore, the analysis therein is independent of the TLCM analysis here. According to their characterization, the planet orbits a supersolar star (mass: $1.14 \pm 0.08$ solar mass, radius: $1.02 \pm 0.05$ solar radii, and $T_{\text {eff }}=5880 \pm 90 \mathrm{~K}$ with a metallicity of $[\mathrm{Fe} / \mathrm{H}]=0.14 \pm 0.12$ ). The planet has relatively long period among 
Table 5. Comparison of the LC solutions of CoRoT-20b obtained by this study and by Deleuil et al. (2012). RN: red noise. Notations as usual. Eccentricity and argument of periastron were calculated from $\sqrt{e} \sin \omega$ and $\sqrt{e} \cos \omega$, respectively. Inclination is derived from impact/conjunction parameters. The inclusion of the red noise did not yield different fits and the red noise is negligible in this LC. The column difference gives the difference between the solutions of Deleuil et al. (2012) and the one obtained by TLCM without red noise. The $\sigma$ is defined as for Table 4 .

\begin{tabular}{|c|c|c|c|c|}
\hline Parameter & Deleuil et al. (2012) & TLCM, with RN & TLCM, without RN & Difference \\
\hline Orbital period & $9.24285 \pm 0.00030 \mathrm{~d}$ & $9.24285 \mathrm{~d}$ (fixed) & $9.24285 \mathrm{~d}$ (fixed) & - \\
\hline Transit epoch & BJD 55266.0001 \pm 0.0014 & $55266.0010 \pm 0.0017$ & BJD $55266.0009 \pm 0.0016$ & $0.4 \sigma$ \\
\hline$e \sin \omega$ & $0.468 \pm 0.017$ & - & - & - \\
\hline$e \cos \omega$ & $0.312 \pm 0.022$ & - & - & - \\
\hline$\sqrt{e} \sin \omega$ & - & $0.630 \pm 0.042$ & $0.627 \pm 0.024$ & - \\
\hline$\sqrt{e} \cos \omega$ & - & $0.421 \pm 0.050$ & $0.425 \pm 0.028$ & - \\
\hline $\mathrm{e}$ & $0.562 \pm 0.013$ & $0.574 \pm 0.035$ & $0.574 \pm 0.020$ & $0.5 \sigma$ \\
\hline$\omega[\operatorname{deg}]$ & $56.3_{-2.3}^{+2.4}$ & $56.3 \pm 3.6$ & $55.9 \pm 2.0$ & $0.1 \sigma$ \\
\hline $\mathrm{K}$ & $454 \pm 9 \mathrm{~m} \mathrm{~s}^{-1}$ & $458 \pm 19 \mathrm{~m} \mathrm{~s}^{-1}$ & $458 \pm 11 \mathrm{~m} \mathrm{~s}^{-1}$ & $0.2 \sigma$ \\
\hline$V_{\gamma}$ & $60.623 \pm 0.006 \mathrm{~km} \mathrm{~s}^{-1}$ & $60.618 \pm 0.014 \mathrm{~km} \mathrm{~s}^{-1}$ & $60.619 \pm 0.008 \mathrm{~km} \mathrm{~s}^{-1}$ & $0.5 \sigma$ \\
\hline \multicolumn{5}{|l|}{ RV offsets: } \\
\hline HARPS-SOPHIE & $93 \pm 11 \mathrm{~ms}^{-1}$ & $85 \pm 35 \mathrm{~m} \mathrm{~s}^{-1}$ & $87 \pm 20 \mathrm{~m} \mathrm{~s}^{-1}$ & $0.3 \sigma$ \\
\hline SOPHIE-FIES & $163 \pm 20 \mathrm{~ms}^{-1}$ & $166 \pm 37 \mathrm{~m} \mathrm{~s}^{-1}$ & $168 \pm 21 \mathrm{~m} \mathrm{~s}^{-1}$ & $0.2 \sigma$ \\
\hline$R_{\mathrm{p}} / R_{\mathrm{S}}$ & $0.0842 \pm 0.0017$ & $0.0898 \pm 0.0049$ & $0.0865 \pm 0.0052$ & $0.4 \sigma$ \\
\hline$b^{\prime}$ & $0.26 \pm 0.08$ & $0.01 \pm 0.31$ & $0.03 \pm 0.56$ & $0.4 \sigma$ \\
\hline$a / R_{\mathrm{S}}$ & $18.95_{-0.73}^{+0.63}$ & $17.77 \pm 2.69$ & $18.15 \pm 2.57$ & $0.3 \sigma$ \\
\hline$p_{0}$ & - & $425 \pm 228 \mathrm{ppm}$ & $275 \pm 139 \mathrm{ppm}$ & - \\
\hline inclination & $88.21 \pm 0.53 \mathrm{deg}$ & $89.90_{-3.7}^{+0.10}$ & $89.7_{-3.3}^{+0.2} \mathrm{deg}$ & $0.4 \sigma$ \\
\hline$\sigma_{r}$ & - & $2225 \pm 121$ & - & - \\
\hline$\sigma_{w}$ & - & $2363 \pm 228$ & - & - \\
\hline Stellar density & $1.51_{-0.17}^{+0.15} \mathrm{~g} \mathrm{~cm}^{-3}$ & $1.65 \pm 0.68 \mathrm{~g} \mathrm{~cm}^{-3}$ & $1.35 \pm 0.58 \mathrm{~g} \mathrm{~cm}^{-3}$ & $0.3 \sigma$ \\
\hline$\left(M_{\mathrm{star}} / \mathrm{M}_{\odot}\right)^{1 / 3} /\left(R_{\mathrm{star}} / \mathrm{R}_{\odot}\right)$ & $1.022_{-0.039}^{+0.034}$ & $1.05 \pm 0.14$ & $0.98 \pm 0.14$ & $0.3 \sigma$ \\
\hline$M_{\mathrm{star}} / \mathrm{M}_{\odot}$ & $1.14 \pm 0.08$ & $1.02 \pm 0.19$ & $1.00 \pm 0.05$ & $1.5 \sigma$ \\
\hline$R_{\mathrm{star}} / \mathrm{R}_{\odot}$ & $1.02 \pm 0.05$ & $0.95 \pm 0.06$ & $0.96 \pm 0.16$ & $0.4 \sigma$ \\
\hline$M_{\text {planet }} / M_{\text {Jup }}$ & $4.24 \pm 0.23$ & $3.75 \pm 0.46$ & $3.77 \pm 0.43$ & $1.0 \sigma$ \\
\hline$R_{\text {planet }} / M_{\text {Jup }}$ & $0.84 \pm 0.04$ & $0.90 \pm 0.10$ & $0.85 \pm 0.09$ & $0.1 \sigma$ \\
\hline
\end{tabular}

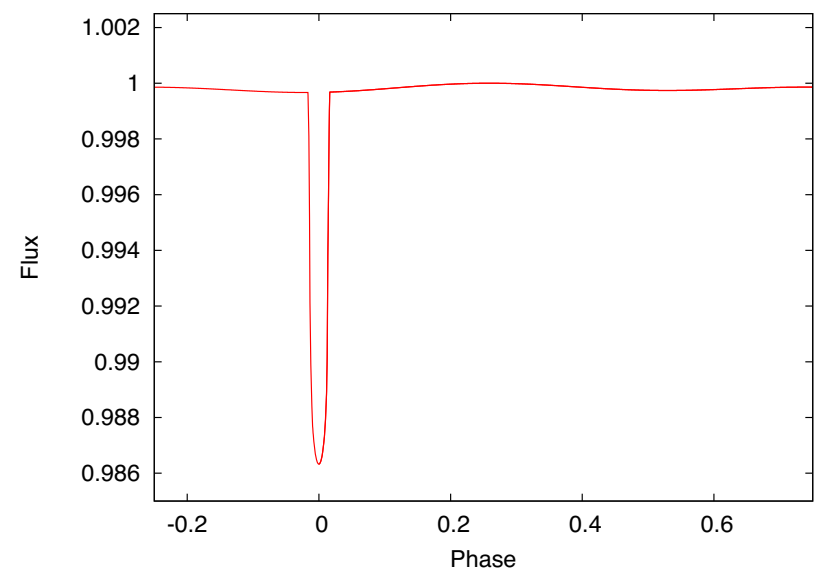

Figure 15. The full LC with all LC components of both components totalized. The parameters in Table 6 were used to produce this plot.

the transiting exoplanets $(P=9.24285 \mathrm{~d})$ and has a high eccentricity $(e=0.56)$.

The same RV data were used for the present fit what Deleuil et al. (2012) presented. The CoRoT LC was downloaded from the CoRot data archive. ${ }^{13}$ The white flux data were used, only with flags 0 (valid

\footnotetext{
${ }^{13} \mathrm{http} / / /$ idoc-corot.ias.u-psud.fr/sitools/client-user/COROT_N2_PUBLIC_D ATA/project-index.html
}

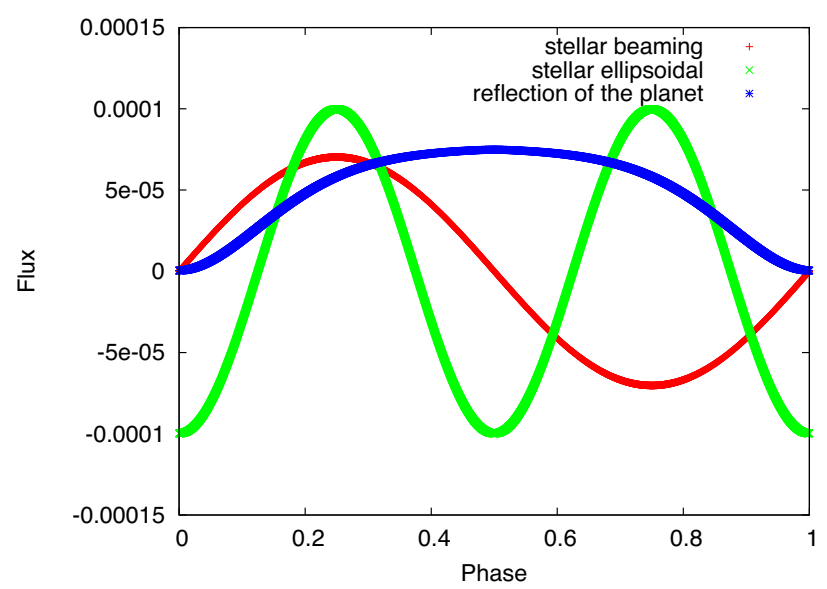

Figure 16. A zoom to the different components of the model presented in Fig. 15. The parameters reported in Table 6 were used to produce this curve. The red curve and the green curve are the beaming and ellipsoidal effects of the host star, respectively, and the blue curve is the reflection effect that occurred in the planetary companion. The $x$-axis is phase, and the $y$-axis is flux.

measurement) or 1 (outlier, but no reason to remove it). All observed transit events were cut and the vicinity of each transit ( 3 times of the transit length) was fitted by a parabola using the out-of-transit points only, and then all flux values - including in-transit points too - in these windows were divided by the corresponding parabola. Then the LC was folded with the ephemeris given in Deleuil et al. (2012). 


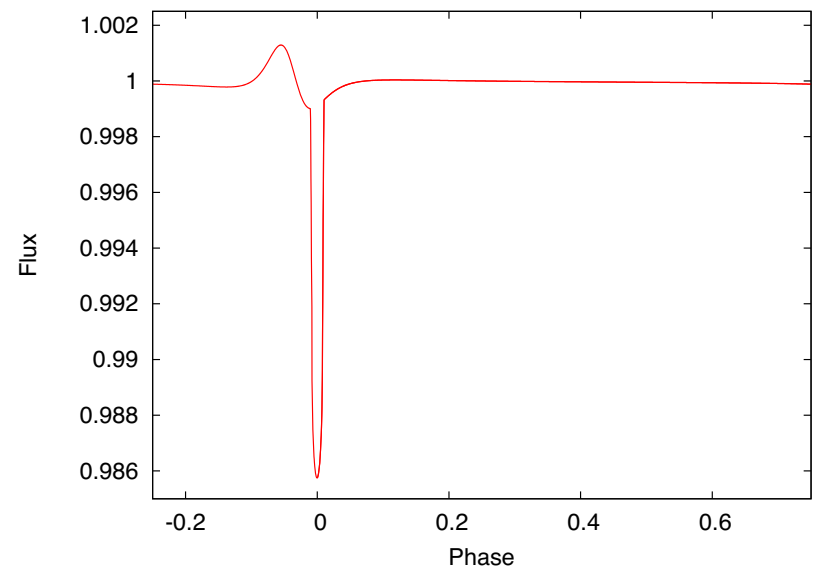

Figure 17. The full LC with all LC components summed up. The parameters in Table 6 were used to produce this plot, but an eccentric orbit was set with $e \sin \omega=0.3$ and $e \cos \omega=0.5$. The bump before the transit is the so-called periastron effect or 'heart-beat' binarity effect.

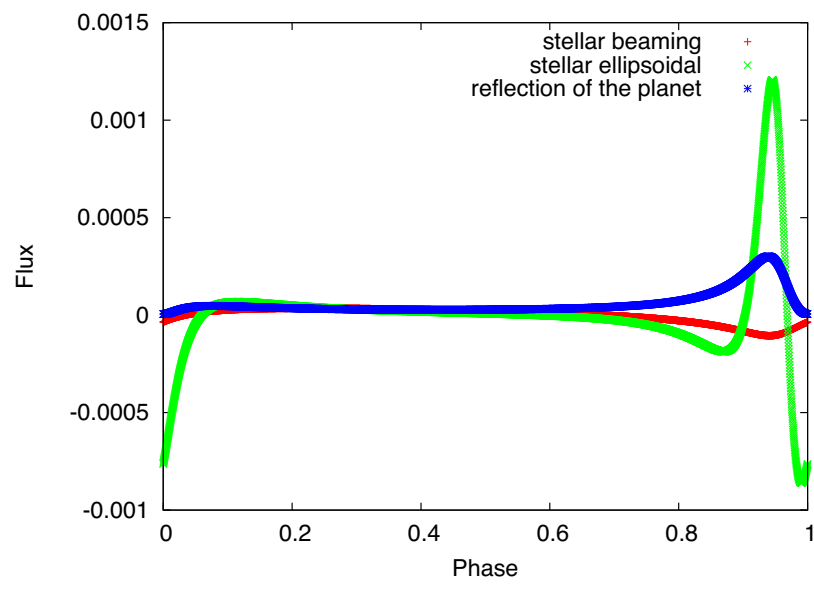

Figure 18. A zoom to the different components of the model presented in Fig. 17.

Table 6. Parameters used for producing Figs 15-18. A circular orbit was set. Ellipsoidal coefficients not reported in the table were set to zero and the stellar albedo was zero.

\begin{tabular}{lc}
\hline Parameter & Value \\
\hline Planetary albedo & 0.3 \\
$\sigma_{w}=\sigma_{r}$ & 0.0 \\
$a / R_{\mathrm{S}}$ & 9.0 \\
$R_{\mathrm{p}} / R_{\mathrm{S}}$ & 0.1115 \\
$b^{\prime}$ & 0.6 \\
$u_{+, s}$ & 0.5 \\
$u_{-, s}$ & 0.2 \\
$l_{3}$ & 0.0 \\
$\mathrm{E}$ & 0.0 \\
$\mathrm{P}$ & 3.0 \\
$V_{\gamma}$ & 0.0 \\
$K_{1}$ & $5.0 \mathrm{~km} \mathrm{~s}$ \\
$A_{2}$ & 0.5 \\
$a_{i 2}$ & $100 \mathrm{ppm}$ \\
$f$ & $0 \mathrm{ppm}$ \\
\hline
\end{tabular}

Table 7. Comparison of the RV amplitudes obtained from separate RV fits and from photometric beaming amplitude by Faigler et al. (2015), and photometric beaming amplitude by TLCM.

\begin{tabular}{lrrr}
\hline System & \multicolumn{1}{c}{$K_{\text {RV }}$} & \multicolumn{1}{c}{$K_{\text {beaming }}$} & $K_{\text {TLCM, phot }}$ \\
\hline KIC 2851474 & $16.81 \pm 0.9$ & $15.4 \pm 1.9$ & $20.4 \pm 0.5$ \\
KIC 4161474 & $23.7 \pm 1.5$ & $9.1 \pm 1.1$ & $16.6 \pm 3.0$ \\
KIC 6515722 & $16.8 \pm 1.7$ & $17.4 \pm 1.2$ & $17.4 \pm 0.5$ \\
KIC 9285587 & $19.8 \pm 1.4$ & $15.9 \pm 2.4$ & $19.6 \pm 1.3$ \\
\hline
\end{tabular}

A joint fit of the LC and RV data was then performed. The results are shown in Figs 13 and 14 and in Table 5. A good agreement was found by the present results and those of Deleuil et al. (2012). All the differences between the results of Deleuil et al. (2012) and of this study are much smaller than the respective error bars, so this validates the code again.

\subsection{Out-of-transit variations}

In Figs 15-18, some examples are shown for the out-of-transit variation calculations. Table 6 presents the parameters used to produce these figures. Figs 15 and 16 present circular orbit cases. Figs 17 and 18 show examples with an eccentric orbit $(e \sin \omega=0.3$, $e \cos \omega=0.5$, so $e=0.7823$ and $\omega=30.96^{\circ}$ ).

Four eclipsing binaries observed by Kepler were modelled with this capacity of the code in order to check TLCM. The binaries are selected from Faigler et al. (2015) because they have RV curves observed too. This provide a unique opportunity to compare the observed RV amplitudes to the estimates based on the beaming effect in the LC.

The LC data were downloaded from the public Kepler MAST archive and periods were taken from Faigler et al. (2015). Every orbital cycle was divided by its median value, and then the cycles were folded and binned in the orbital phase space to 500 data points. These LCs were fitted. Effective temperatures of the primaries were also taken from Faigler et al. (2015) and following them I set solar metallicity for these four binaries.

The beaming, reflection, and ellipsoidal effects beyond the transits and occultations were included.

The LC solutions are presented in Tables 7 and 8 . Table 7 compares the measured RV amplitudes ( $\left.K_{\mathrm{RV}}\right)$ to the RV amplitude estimates from beaming effect done by Faigler et al. (2015), denoted by $K_{\text {beaming }}$ in that table, and also compares them to the ones TLCM provided

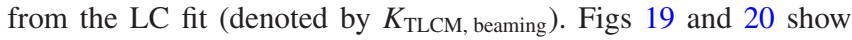
examples of these fits.

The total sum of the differences in the RV amplitudes measured from the RV curve and from the LC curve is $20.51 \mathrm{~km} \mathrm{~s}^{-1}$ by Faigler et al. (2015), while it is just $11.04 \mathrm{~km} \mathrm{~s}^{-1}$ by TLCM. In average, the difference between the RV and beaming-based RV amplitudes is $5.12 \mathrm{~km} \mathrm{~s}^{-1}$ by Faigler et al. (2015) and $2.77 \mathrm{~km} \mathrm{~s}^{-1}$ by TLCM, so I have 84 per cent improvement in the beaming-based RV amplitude estimates in these four cases relative to the previous study.

\section{SUMMARY}

In this paper, I presented TLCM, a computer code to simulate, to analyse, and to fit LC and RV curves of detached eclipsing binaries and transiting exoplanets. It utilizes the Mandel \& Agol (2002) model and routines to describe the transits and occultations themselves, but it is extended to include the beaming, ellipsoidal, and reflection effects of the primary and secondary objects, which allows the user to analyse the phase curves or out-of-transit variations 
Table 8. LC solutions of KIC 2851474, 4169521, 6515722, and 9285587. LC data are taken from the public Kepler MAST archive, and periods are taken from Faigler et al. (2015). All orbits were assumed to be circular.

\begin{tabular}{lcccc}
\hline Parameter & KIC2851474 & KIC4169521 & KIC6515722 & KIC9285587 \\
\hline Orbital period [days] & 2.7682925 & 1.1725557000 & 3.8170428 & 1.8119579 \\
$K_{\text {beaming }}[\mathrm{km} / \mathrm{s}]$ & $20.4 \pm 0.5$ & $16.6 \pm 0.6$ & $17.4 \pm 0.5$ & $19.6 \pm 1.3$ \\
$R_{2} / R_{1}$ & $0.0103 \pm 0.0004$ & $0.0446 \pm 0.0010$ & $0.0094 \pm 0.0006$ & $0.0136 \pm 0.0034$ \\
$b$ & $0.35 \pm 0.03$ & $0.94 \pm 0.05$ & $0.49 \pm 0.03$ & $0.59 \pm 0.06$ \\
$a / R_{\mathrm{s}}$ & $3.98 \pm 0.11$ & $2.1 \pm 0.1$ & $6.06 \pm 0.11$ & $3.22 \pm 0.11$ \\
Stellar density $\left[\mathrm{g} \mathrm{cm}^{-3}\right]$ & $155 \pm 10$ & $133 \pm 10$ & $288 \pm 15$ & $193 \pm 20$ \\
$M_{1} / \mathrm{M}_{\odot}$ & $1.99 \pm 0.12$ & $2.10 \pm 0.20$ & $2.0 \pm 0.17$ & $1.74 \pm 0.53$ \\
$R_{1} / \mathrm{R}_{\odot}$ & $2.72 \pm 0.06$ & $2.87 \pm 0.09$ & $2.21 \pm 0.08$ & $2.41 \pm 0.24$ \\
$M_{2} / \mathrm{M}_{\odot}$ & $0.23 \pm 0.02$ & $0.16 \pm 0.03$ & $0.22 \pm 0.02$ & $0.18 \pm 0.04$ \\
$R_{2} / \mathrm{M}_{\odot}$ & $0.028 \pm 0.002$ & $0.13 \pm 0.01$ & $0.021 \pm 0.002$ & $0.033 \pm 0.009$ \\
\hline
\end{tabular}
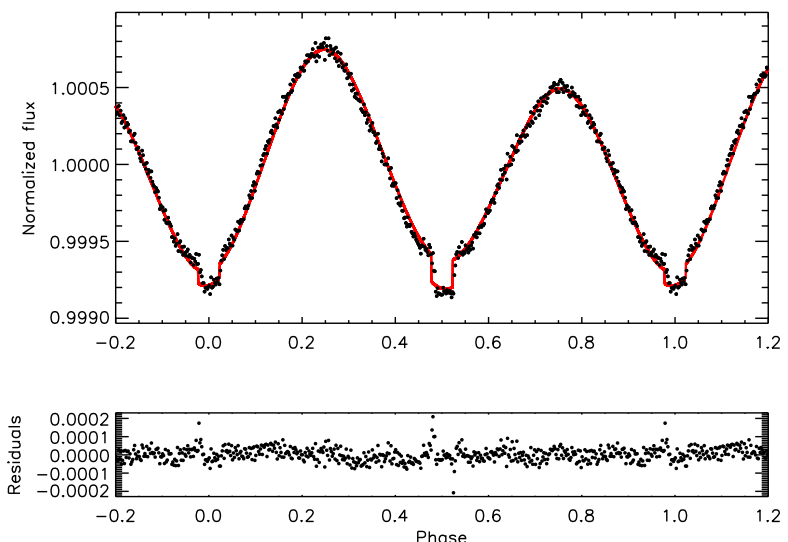

Figure 19. The LC solution of KIC 6515722. The lower panel shows the residuals while the upper panel does the observed points (black dots) and the fit (red curve).
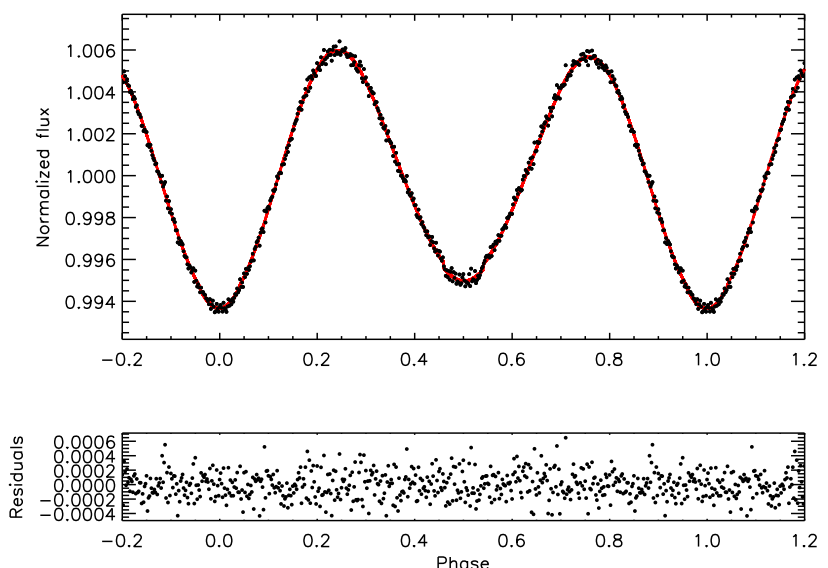

Figure 20. The LC solution of KIC 9285587. The lower panel shows the residuals while the upper panel does the observed points (black dots) and the fit (red curve).

of an exoplanet or a companion star. The effect of binning is also included, and the contamination is considered, which can be owing to an unresolved, physically bounded or not bounded companion, a background galaxy, stellar spots and faculae, background-subtraction problem, etc. Several other auxiliary quantities are used to fit the LCs, like baseline corrections, shifts in the flux level or in the epoch. A red noise model based on the wavelet models and routines of Carter \& Winn (2009) is included.
The code is able to perform a joint $\mathrm{LC}+\mathrm{RV}$ fit and a simplified $\mathrm{RM}$ effect is included.

Various outputs - ASCII files and figures - are produced by TLCM, which can be used directly in the publications or the user can make his/her own figures. The absolute dimensions - mass and radius of the primary and the secondary - are calculated for SB2 binaries in a model-independent way. When only one RV curve is available (SB1 binaries), then the primary's mass and radius are estimated from the temperature and metallicity of the primary and from the mean stellar density measured from the LC. When no RV measurement is available, then the beaming effect is used for this purpose. When the quality of the LC does not allow the fit of the beaming effect, then only the radii of the two components are estimated like in the case of SB1 systems. For this estimate, TLCM uses the analytic expressions of Hurley et al. (2000) or the empirical calculations of Soutworth (2011).

Improvements in the understanding of the beaming effect are presented in this study, which includes a more physical description of the reflection and ellipsoidal effects that interfere with the beaming effects. This yields a more perfect agreement between the RV amplitudes measured from the RV curves and the ones obtained from the LCs. However, further improvements of TLCM include multicolour, very precise LCs, because then the reflection and the beaming effect could be monitored on different wavelengths and their effects could be separated in a much easier way.

I also showed that replacing the wavelength integrated limbdarkening coefficients with a passband-specific value has a ca. 16 per cent variation in the amplitude of the RM effect. This seems to be negligible today but it might be taken when more precise data of the ESPRESSO instrument or ELT will be analysed.

I also called the attention that RV drifts cause beaming effect as well. Such a drift in K2-99 system may lead to a $10 \mathrm{ppm} \mathrm{yr}^{-1}$ variation in the mean brightness of the host star, which seems to be observable with PLATO.

TLCM can be downloaded from the homepage mentioned in Section 7.

The code is freely available and runs under IDL and free GDL, under the Unix, Linux, and Windows platforms. It can be run from command line or an $\mathrm{X}$-window is also available, depending on the user's choice.

\section{ACKNOWLEDGEMENTS}

The author thanks DFG Research Unit 2440: 'Matter Under Planetary Interior Conditions: High Pressure, Planetary, and Plasma Physics' for the support. He also thanks the Hungarian National Research, Development and Innovation Office, for the NKFIH-OTKA KH- 
130372 grant and the German Priority Program 1992: 'Mass-radius diversity of exoplanets' RA714/14-1. The continuous pressing of several colleagues, especially Drs. Juan Cabrera, Péter Klagyivik, and Prof. Heike Rauer among others, which targeted the author to publish TLCM and its detailed description, is noted and acknowledged. The help of Drs. Alexis Smith and Philipp Eigmüller and Mrs Judith Korth, who tested TLCM extensively and corrected my English, is also acknowledged. Comments of the referee (J. Eastman) are also acknowledged. This paper was submitted on the 400th anniversary of the discovery of Kepler's third law.

\section{REFERENCES}

Albrecht S., 2012, in Richards M. T., Hubeny I., eds, Proc. IAU Symp. 282, From Interacting Binaries to Exoplanets: Essential Modeling Tools. Kluwer, Dordrecht, p. 379

Alonso R. et al., 2009, A\&A, 506, 353

Alonso R., Deeg H. J., Kabath P., Rabus M., 2010, AJ, 139, 1481

Andrae R. et al., 2018, A\&A, 616, A8

Angerhausen D., DeLarme E., Morse J. A., 2015, PASP, 127, 1113

Attia A.-F., Mahmoud E., Shahin H. I., Osman A. M., 2009, New Astron., 14,285

Bakos G. et al., 2009, in Pont F., Sasselov D. D., Holman M. J., eds, Proc. IAU Symp. 253, Transiting Planets. Kluwer, Dordrecht, p. 354

Bakos G. Á. et al., 2010, ApJ, 710, 1724

Barge P. et al., 2008, A\&A, 482, L17

Barragán O., Gandolfi D., Antoniciello G., 2019, MNRAS, 482, 1017

Barros S. C. C. et al., 2014, A\&A, 569, A74

Bayliss D. et al., 2018, MNRAS, 475, 4467

Birkby J. et al., 2012, MNRAS, 426, 1507

Bloemen S. et al., 2011, MNRAS, 410, 1787

Bonomo A. S. et al., 2017, A\&A, 602, A107

Bordé P. et al., 2010, A\&A, 520, A66

Borkovits T., Csizmadia S., Forgács-Dajka E., Hegedüs T., 2011, A\&A, 528, A53

Bouchy F. et al., 2011, A\&A, 525, A68

Broeg C. et al., 2013, EPJWC 4703005

Brown T. M., Charbonneau D., Gilliland R. L., Noyes R. W., Burrows A., 2001, ApJ, 552, 699

Bruno G. et al., 2015, A\&A, 573, A124

Cabrera J. et al., 2010, A\&A, 522, A110

Cabrera J. et al., 2014, ApJ, 781, 18

Cabrera J. et al., 2015, A\&A, 579, A36

Cantó J., Curiel S., Martínez-Gómez E., 2009, A\&A, 501, 1259

Carter J. A., Winn J. N., 2009, ApJ, 704, 51

Charbonneau P., 1995, ApJS, 101, 309

Charbonneau D., Brown T. M., Latham D. W., Mayor M., 2000, ApJ, 529, L45

Charbonneau D. et al., 2009, Nature, 462, 891

Chen B.-Q. et al., 2019, MNRAS, 483, 4277

Claret A., 2004, A\&A, 424, 919

Clausen J. V., Bruntt H., Claret A., Larsen A., Andersen J., Nordström B., Giménez A., 2009, A\&A, 502, 253

Coughlin J. L., López-Morales M., 2012, AJ, 143, 39

Croll B., 2006, PASP, 118, 1351

Csizmadia S., Pasternacki T., 2013, in Chavez M., Bertone E., Vega O., De la Luz V., eds, ASP Conf. Ser. Vol. 472, New Quests in Stellar Astrophysics III: A Panchromatic View of Solar-Like Stars, With and Without Planets. Astron. Soc. Pac., San Francisco, p. 147

Csizmadia S. et al., 2010, A\&A, 510, A94

Csizmadia S. et al., 2011, A\&A, 531, A41

Csizmadia S. et al., 2013, A\&A, 549, A9

Csizmadia S. et al., 2015, A\&A, 584, A13

Csizmadia S., Hellard H., Smith A. M. S., 2019, A\&A, 623, A45

Deeg H., 2018, in Deeg H., Belmonte A., eds, Handbook of Exoplanets, paper E13. Springer Int. Publ. AG, Berlin
Deeg H. J. et al., 2010, Nature, 464, 384

Deleuil M. et al., 2012, A\&A, 538, A145

Deming D., Seager S., 2017, J. Geophys. Res.: Planets, 122, 53

Dressing C. D., Newton E. R., Schlieder J. E., Charbonneau D., Knutson H. A., Vanderburg A., Sinukoff E., 2017, ApJ, 836, 167

Dunkley J., Bucher M., Ferreira P. G., Moodley K., Skordis C., 2005, MNRAS, 356, 925

Eastman J., Gaudi B. S., Agol E., 2013, PASP, 125, 83

Eigmüller P. et al., 2016, AJ, 151, 84

Eigmüller P. et al., 2017, AJ, 153, 130

Eigmüller P. et al., 2018, MNRAS, 480, 3864

Enoch B., Collier Cameron A., Parley N. R., Hebb L., 2010, A\&A, 516, A33

Espinoza N., Jordán A., 2015, MNRAS, 450, 1879

Faigler S., Kull I., Mazeh T., Kiefer F., Latham D. W., Bloemen S., 2015, ApJ, 815, 26

Ford E. B., 2006, ApJ, 642, 505

Fridlund M. et al., 2010, A\&A, 512, A14

Gandolfi D. et al., 2010, A\&A, 524, A55

Gandolfi D. et al., 2013, A\&A, 557, A74

Gao X. Z., Govindasamy V., Xu H., Wang X., Zenger K., 2015, Comput. Intell. Neurosci., 258491

Geem Z. W., 2009, Music-Inspired Harmony Search Algorithm, Theory and Applications - Studies in Computational Intelligence 191. SpringerVerlag, Berlin

Geem Z. W., Kim J. H., Lonatathan G. V., 2001, Simulation, 76, 60

Gimenez A., 2006a, A\&A, 450, 1231

Gimenez A., 2006b, ApJ, 650, 408

Gimenez A., Garcia-Pelayo J. M., 1983, Ap\&SS, 92, 203

Guenther E. et al., 2012, A\&A, 538, A136

Haywood R. D. et al., 2014, MNRAS, 443, 2517

Hébrard G. et al., 2011, A\&A, 533, A130

Henry G. W., Marcy G. W., Butler R. P., Vogt S. S., 2000, ApJ, 529, L41

Holman M. J., Murray N. W., 2005, Science, 307, 1288

Huber K. F., Czesla S., Schmitt J. H. M. M., 2017, A\&A, 597, A113

Hurley J. R., Pols O. R., Tout Ch. A., 2000, MNRAS, 315, 543

Jackson R. J. et al., 2016, A\&A, 586, A52

Ji Y., Banks T., Budding E., Rhodes M. D., 2017, Ap\&SS, 362, 112

Kane S. R., Gelino D. M., 2011, ApJ, 729, 74

Kipping D. M., 2010, MNRAS, 408, 1758

Klagyivik P. et al., 2016, AJ, 151, 110

Konacki M., Torres G., Jha S., Sasselov D. D., 2003, Nature, 421, 507

Kopal Z., 1959, Close Binary Systems (The International Astrophysics Series). Chapman \& Hall, London

Korth J. et al., 2019, MNRAS, 482, 1807

Lallement R., Babusiaux C., Vergely J. L., Katz D., Arenou F., Valette B., Hottier C., Capitanio L., 2019, A\&A, 625, A135

Lastennet E., Valls-Gabaud D., 2002, A\&A, 396, 551

Loeb A., Gaudi B. S., 2003, ApJ, 588, L117

Mallonn M. et al., 2015, A\&A, 583, A138

Mandel K., Agol E., 2002, ApJ, 580, L171

Mann A. W., Feiden G. A., Gaidos E., Boyajian T., von Braun K., 2015, ApJ, 804, 64

Martynov D. Y., 1973, in Tsessevich V. P., ed., Eclipsing Variable Stars. IPST Astroph. Libr., Jerusalem, p. 313

Mazeh T., Faigler S., 2010, A\&A, 521, L59

Metcalfe T. S., 1999, AJ, 117, 2503

Milone E. F., Kallrath J., 2009, Eclipsing Binary Stars: Modelling and Analysis. Springer-Verlag, New York

Munari U., Sordo R., Castelli F., Zwitter T., 2005, A\&A, 442, 1127

Neilson H. R., Lester J. B., 2013, A\&A, 556, A86

Ollivier M. et al., 2012, A\&A, 541, A149

Pätzold M. et al., 2012, A\&A, 545, A6

Pál A., 2008, MNRAS, 390, 281

Parviainen H., Deeg H. J., Belmonte J. A., 2013, A\&A, 550, A67

Pepper J. et al., 2007, PASP, 119, 923

Pollacco D. L. et al., 2006, PASP, 118, 1407

Prša A., Zwitter T., 2005, ApJ, 628, 426 
Raetz S. et al., 2015, MNRAS, 451, 4139

Rauer H. et al., 2009, A\&A, 506, 281

Rauer H. et al., 2010, AJ, 139, 53

Rauer H. et al., 2014, Exp. Astron., 38, 249

Rouan D. et al., 2012, A\&A, 537, A54

Russell H. N., 1912, ApJ, 35, 315

Schneider J., Dedieu C., Le Sidaner P., Savalle R., Zolotukhin I., 2011, A\&A, 532, A79

Sedaghati E. et al., 2016, A\&A, 596, A47

Sedaghati E., Boffin H. M. J., Delrez L., Gillon M., Csizmadia S., Smith A. M. S., Rauer H., 2017, MNRAS, 468, 3123

Silva-Valio A., Lanza A. F., Alonso R., Barge P., 2010, A\&A, 510, A25

Smith A. M. S. et al., 2017, MNRAS, 464, 2708

Smith A. M. S. et al., 2019, Acta Astron., 69, 135

Snellen I. A. G., de Mooij E. J. W., Albrecht S., 2009, Nature, 459, 543

Southworth J., 2011, MNRAS, 417, 2166

Southworth J., 2012, MNRAS, 426, 1291

Southworth J., 2013, A\&A, 557, A119

Southworth J., 2014, in Haghighipour N., ed., Proc. IAU Symp. 293, Formation, Detection, and Characterization of Extrasolar Habitable Planets. Kluwer, Dordrecht, p. 423

Sullivan P. W. et al., 2015, ApJ, 809, 77

Sullivan P. W. et al., 2017, ApJ, 837, 99

Torres G., Andersen J., Giménez A., 2010, A\&AR, 18, 67

Torres G., Fischer D. A., Sozzetti A., Buchhave L. A., Winn J. N., Holman M. J., Carter J. A., 2012, ApJ, 757, 161

Vida K., Oláh K., Kôvári Z., Korhonen H., Bartus J., Hurta Z., Posztobányi K., 2009, A\&A, 504, 1021

Weiss L. M., Marcy G. W., 2014, ApJ, 783, L6

West R. G. et al., 2016, The Messenger, 165, 10

Wilson R. E., 1979, ApJ, 234, 1054

Wilson R. E., 1990, ApJ, 356, 613

Wilson R. E., 1994, PASP, 106, 921

Wilson R. E., 2008, ApJ, 672, 575

Wilson R. E., Biermann P., 1976, A\&A, 48, 349

Wilson R. E., Devinney E. J., 1971, ApJ, 166, 605

Winn J. N., 2010, preprint (arXiv:1001.2010)

Zakamska N. L., Pan M., Ford E. B., 2011, MNRAS, 410, 1895

\section{APPENDIX A: RELIABILITY OF THE DERIVED PARAMETERS}

In this Appendix, I discuss the reliability of the derived stellar and planetary parameters. This reliability does not depend on TLCM or on any other modelling code, but mostly does on the input stellar physics.

Planet-to-star radius ratio:

The reliability of the derived planet-to-star radius ratio in TLCM and in other codes is mostly affected by limb-darkening and photometric noise as well as stellar noise. This can be seen from the basic expression of the transit depth:

$d=k^{2} L_{\mathrm{D}}$,

where $d$ is the flux loss due to transit, $k$ is the planet-to-star radius ratio as before, and $L_{\mathrm{D}}$ is the limb darkening function. After logarithmic differentiation, I have

$\frac{\Delta k}{k}=\frac{\sigma}{2}+\frac{\sigma}{d}+\frac{1}{2} \frac{\Delta L_{\mathrm{D}}}{L_{\mathrm{D}}}$

(see Appendix B). Here $\Delta$ denotes the expected error bar and $\sigma$ is the white noise level of the LC averaged for one transit duration. (Red noise is not counted in this estimation.) The first two terms on r.h.s can be decreased down to the negligible range by using better quality photometric data and/or observing deeper transits. However, the third factor depends on our knowledge of limb darkening. As discussed in Csizmadia et al. (2013), three factors have an impact on the last term in equation (A2):

(i) uncertainties of input stellar parameters,

(ii) uncertainties of theoretical limb-darkening tables,

(iii) effect of stellar activity on limb darkening.

Considering the first point, the present-day usual uncertainties in $T_{\text {eff }}$ and $\log g-$ on which the limb-darkening coefficients depend most after the wavelength of the observation - do not allow us to predict the limb-darkening coefficients better than \pm 0.02 in absolute value. For example, in the case of CoRot-1b, Barge et al. (2008) gave $5850 \mathrm{~K}$ for the host star effective surface temperature, while Torres et al. (2012) reported $6298 \mathrm{~K}$, which led to stellar masses of 0.96 solar masses reported by Barge et al. (2008) and 1.11 solar masses by Torres et al. (2012). Such differences, of course, are reflected in the planetary mass, radius, and density values. For the host star of K2-60b, three different codes and teams presented three different effective surface temperatures from the same spectral observations, which differed by $275 \mathrm{~K}$. This corresponds to a $3.3 \sigma$ difference if I take the $85 \mathrm{~K}$ error bar of one team or $2.4 \sigma$ if I use the other one (Eigmüller et al. 2017). Such examples tell us that one has to be careful with stellar parameters and uncertainties. To judge better the systematic shifts in these parameters, a comparison of works by different teams is recommended. The aforementioned differences in stellar parameters may cause a few per cent uncertainty in the values of the limb-darkening coefficients if one takes them from theoretical tables and the errors are propagated to the tables, and also 1-3 per cent error in the planet-to-star radius ratio (cf. equation 1 of Csizmadia et al. 2013).

The reliability of the different theoretical limb-darkening tables was discussed by comparing different tables to each other in Csizmadia et al. (2013) and Neilson \& Lester (2013). The former study showed that limb-darkening uncertainties can cause errors of up to 40 per cent in the derived planetary radii in certain temperature ranges. It is not clear whether fixing or fitting these parameters is the better approach (Csizmadia et al. 2013, Espinoza \& Jordán 2015). Csizmadia et al. (2013) produced synthetic data with white noise and modelled them with TLCM, while fitting the limb-darkening coefficients. They found (their fig. 4) that one can measure well the planet-to-star radius ratio with 1-2 percent accuracy if the transit depth/photometric noise ratio is greater than 3 , and the number of in-transit data points is at least 500 .

Planetary radii after scaling with $R_{\mathrm{s}}$ :

The most important stellar parameter in transit LC analysis is the stellar radius. The most precise stellar radii can be obtained from analysis of double-lined eclipsing binaries and from interferometric measurements, but the latter suffers from unknown distances. ${ }^{14}$ Clausen et al. (2009, and references therein) called attention to the fact that stars smaller than our Sun (down to K5V stars) are 10 percent smaller (at 1 percent measurement accuracy level) in radius than those predicted by stellar models for the given mass. Torres et al. (2010) collected those 190 stars in eclipsing systems that have better radius values than 3 percent. They noticed that several parameter ranges are not well sampled (especially above 10 and below 0.6 solar masses). Their comparison of observations to theoretical stellar models and isochrones revealed that better stellar models are needed for stars below 1 solar mass. Additionally, for stars in the range of 1.1-1.5 solar masses, a more precise description of

\footnotetext{
${ }^{14}$ After the Gaia's data releases, changes in the yield of interferometric measurements are expected.
} 
overshooting and diffusion is needed for better agreement between models and observations. Dressing et al. (2017) found that cool stars (K3-M4 dwarfs) have a 39 percent larger radius than those predicted from isochrones. Similar studies support these findings (e.g. Vida et al. 2009; Eigmüller et al. 2016, and references therein). These findings were later further confirmed by using the first data release of Gaia measurements: Jackson et al. (2016) highlighted that main-sequence stars in open clusters of age 5-140 Myr have radii bigger by 10 per cent than those predicted by standard evolutionary models (the difference is even larger for pre-MS stars according to them). The reason can be the neglected magnetic field; spots can block 30 per cent of the stellar light or a moderate combination of both. If one observes planets around such stars, then all planetary radii are systematically scaled up by 10 percent, which means 30 per cent systematic error in the density. Birkby et al. (2012) and, by interferometry, Mann et al. (2015) pointed out that the discrepancy between models and observations is even more pronounced for late M-dwarf stars where large, 30-50 per cent radius inflation can be observed compared to standard models. Maybe, the magnetically driven strong stellar activity puffs up these stars over the theoretically expected ones, which do not include magnetic field calculations. Also, strong stellar activity (spot) phenomena can contribute to the observed discrepancies that falsify the effective temperature determination, or convection parameters in the models are not well estimated. In conclusion, there is real doubt on the derived stellar diameters from isochrones. Usually, such systematic errors are not taken into account in planetary radius analysis.

The stellar parameters are functions like $f\left(M, Z, t_{\mathrm{s}}\right)$, where $t_{\mathrm{s}}$ is the age of the star; binarity and mass-loss caused by stellar wind can affect the form of function $f$. The inverse problem of stellar parameter determination might be degenerate: the Vogt-Russel theorem may be violated by real stars, i.e. stars can perhaps occupy the same position on the HR-diagram with different masses and chemical compositions (Lastennet \& Valls-Gabaud 2002, and references therein).

Therefore, the used empirical calibrations by the TLCM code have their own significance. Such calibrations are given in Torres, Andersen \& Gimenez (2010) for $\log g, \log T_{\text {eff }}$, and metallicity. Enoch et al. (2010) and Southworth (2011) give the mass and the radius of the host star as a function of the metallicity, effective temperature, and the mean density of the star (which is measurable from the transit duration). Note that in this case the effect of spots is not additional; because of the empirical nature of the calibration of these relationships, they are inherently included. TLCM uses the calibration of Southworth (2011).

In the future accurate Gaia parallaxes and magnitudes should be included into the parameter determination in order to increase the final star and planet parameter precision.

In some other cases where we have very noisy LCs - down to 1:1 transit depth/photometric noise ratio ${ }^{15}$ - the transit duration cannot be measured and therefore the $a / R_{\mathrm{S}}$ ratio is unknown. Strong stellar activity can increase the difficulties. For example, Barros et al. (2014) found by MCMC analysis that without priors, at a low activity level the $a / R_{\mathrm{S}}$ value of CoRoT-7b converges to $\sim 6$ while at a high activity level it converges to $\sim 2$. When observed stellar $T_{\text {eff }}$ and $\log g$ are used as priors in the fit, $a / R_{\mathrm{s}} \sim 4$ is obtained. In such cases, therefore, strong constraints based on stellar parameters should be used to determine the scaled semimajor axis.

\footnotetext{
${ }^{15}$ In this section, photometric noise is the standard deviation of the constant stellar light under white noise calculated for the same time as the transit length.
}

There is hope that Gaia distances combined with the asteroseismology of PLATO will finally resolve these issues and give us reliable, systematics-free, 1-2 percent reliable stellar radii and masses (Rauer et al. 2014). It is worth mentioning that the relative parameters, like $a / R_{\mathrm{s}}$ and planet-to-star radius ratio, are mostly independent of this problem (at a reasonable noise level), and they can be rescaled any time when more accurate stellar radii are available; therefore, they do not have an impact on the relative parameter determination and LC fits of transit LC codes.

With Gaia DR2, one can get very accurate and precise stellar radii - depending on our knowledge of interstellar absorption and the stellar effective temperature. $a / R_{\mathrm{S}}$ then can be fixed or prioritized in two different ways:

(i) The asteroseismologic mean stellar density provides the mass, and Kepler's third law can be used to calculate $a$ and then one can scale it to $a / R_{\mathrm{S}}$ (cf. equation 59 );

(ii) The mean stellar density can be obtained from the transit parameters, and with or without a stellar isochrone model this can be used to get the stellar mass, and then as before, Kepler's third law can be used to calculate $a$ and then one can scale it to $a / R_{\mathrm{s}}$. The second approach was used in Csizmadia et al. (2015).

\section{APPENDIX B: RADIUS RATIO PRECISION, TRANSIT DEPTH, AND LIMB DARKENING}

Here I derive equation (A2), which tells the relationship between transit depth, limb darkening, and radius ratio precision.

Let $d$ denote the transit depth; $\sigma$ is the photometric white noise average for one transit duration, $F_{\text {oot }}$ is the out-of-transit flux, and $F_{\text {in }}$ is the in-transit flux (stellar variability assumed to be removed).

The transit depth is clearly

$d=F_{\text {oot }}-F_{\text {in }}$

and it is related to the radius ratio $k$ and limb-darkening function $L_{\mathrm{D}}$ as

$d=F_{\mathrm{oot}}-F_{\text {in }}=k^{2} L_{\mathrm{D}} \cdot F_{\mathrm{oot}}$.

$L_{\mathrm{D}}$ depends on the limb-darkening coefficients and the actual skyprojected distance of the two objects. Differentiation gives

$\Delta(d)=2 k \Delta(k) L_{\mathrm{D}} F_{\text {oot }}+k^{2} \Delta\left(L_{\mathrm{D}}\right) F_{\mathrm{oot}}+k^{2} L_{\mathrm{D}} \Delta\left(F_{\text {oot }}\right)$.

In case of Poisson noise, one can write that

$\sigma=\Delta\left(F_{\text {oot }}\right) / F_{\text {oot }}$

and therefore

$\Delta(d)=\Delta\left(F_{\text {oot }}-F_{\text {in }}\right) \approx 2 \Delta\left(F_{\text {oout }}\right)=2 \sigma F_{\text {oot }}$.

Putting this back and rearranging equation (B3), I arrived to

$\frac{\Delta(k)}{k}=\frac{\sigma}{2}+\frac{\sigma}{d}+\frac{\Delta\left(L_{\mathrm{D}}\right)}{L_{\mathrm{D}}}$

Q.E.D.

\section{APPENDIX C: DEFINITION OF CONJUNCTION AND PERIOD VARIATION CAUSED BY PROPER MOTION}

The conjunction occurs when the planet crosses the $X Z$ plane (cf. Fig. 1). When $X>0$, one speaks about occultation while one has a transit when $X<0$. The $X$-axis connects the star and the observer, so when the star and the observer do not change their relative position 
in space, then the transit period is equal to the anomalistic period (the latter one appears in Kepler's third law) and it can be considered as the time elapsed from conjunction to conjunction.

When the star exhibits observable proper motion, then the conjunction line slowly moves in space, which leads to an apparent period increase. This is because the planet must do more than one revolution to cross the conjunction line, so the transit period is longer than the anomalistic period.

One can express this increase as

$P_{\mathrm{t}}=P_{\mathrm{a}}\left(1+\frac{N P_{\mathrm{a}} p}{360^{\circ}}\right)$,

where $P_{\mathrm{t}}$ and $P_{\mathrm{a}}$ are the transit and anomalistic periods in days, respectively, $N$ is the number of revolutions, and $p$ is the proper motion in $\operatorname{deg} \mathrm{d}^{-1}$.

The corresponding $O-C$ value can be obtained via

$O-C_{\text {contribution }}=\frac{N(N-1)}{2} P_{\mathrm{a}} p$.

TRAPPIST-1 has an apsidal motion of the order of 1000 mas $\mathrm{yr}^{-1}$. If it had a $1 \mathrm{~d}$-orbital period planet, then we would observe $12 \mathrm{~s} O-$ $C$ value after 1 yr. However, most of the transit planet host stars have a proper motion that is two orders of magnitude or more smaller, so this effect should be taken into account very rarely.

The RV of the exoplanet causes also a period shift via the Doppler effect, which is characterized as

$P_{\text {shifted }}=P_{\mathrm{a}}\left(1+\frac{V_{\gamma}}{c}\right)$.

However, this causes no period variations until $V_{\gamma}$ is constant in time. However, if there is, for instance, a close companion to the host star, then a correction might sometimes be appropriate.

\section{APPENDIX D: FURTHER NOTES ON EQUATION (44)}

Let us assume that the RV points' uncertainties follow a normal distribution. Then the chance that an RV point has a certain value is

$L_{i}=\frac{1}{\sqrt{2 \pi} \sigma_{i}} \mathrm{e}^{-\frac{\left(R V\left(t_{i}\right)-R V_{\text {model }, i}\right)^{2}}{2 \sigma_{i}^{2}}}$.

The probability of the RV fit is the product of each of the points:

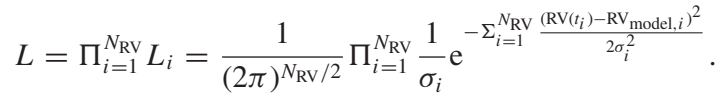

What I have in the exponent is actually the $\chi_{\mathrm{RV}}^{2}$ value of the RV curve. The probability of the joint $\mathrm{RV}+\mathrm{LC}$ fit is the product of the probabilities of the two data sets:

$L_{\text {joint }}=L_{\mathrm{LC}} \times L_{\mathrm{RV}}$

or equivalently,

$-\log L_{\text {joint }}=-\log L_{\mathrm{LC}}-\log L_{\mathrm{RV}}$.

Substituting (D2) into (D4), one gets

$$
\begin{aligned}
-\ln L_{\text {joint }}= & -\ln L_{\mathrm{LC}}+\frac{1}{2}\left(\chi_{\mathrm{RV}, 1}^{2}+\chi_{\mathrm{RV}, 2}^{2}\right. \\
& \left.+\Sigma_{i=1}^{N_{\mathrm{RV}}} \ln \sigma_{\mathrm{RV}, i}+N_{\mathrm{RV}} \ln 2 \pi\right) .
\end{aligned}
$$

With $Q=-\log L_{\text {joint }}$ this is equivalent to equation (44). It inherently contains the assumptions that the RV curve has normally distributed errors.

This paper has been typeset from a $\mathrm{T}_{\mathrm{E}} \mathrm{X} / \mathrm{LT} \mathrm{E} \mathrm{X}$ file prepared by the author. 